USTC-ICTS-19-15

\title{
A New Littlest Seesaw Model
}

\author{
Ping-Tao Chen ${ }^{1 *}$, Gui-Jun Ding ${ }^{1 \dagger}$, Stephen F. King ${ }^{2 \ddagger}$ Cai-Chang Li ${ }^{1,3 \S}$ \\ ${ }^{1}$ Interdisciplinary Center for Theoretical Study and Department of Modern Physics, \\ University of Science and Technology of China, Hefei, Anhui 230026, China \\ ${ }^{2}$ Physics and Astronomy, University of Southampton, Southampton, SO17 1BJ, U.K. \\ ${ }^{3}$ School of Physics, Northwest University, Xi'an 710127, China
}

\begin{abstract}
We propose and discuss a new Littlest Seesaw model, realized in the tri-direct CP approach, in which the couplings of the two right-handed neutrinos to the lepton doublets are proportional to $(0,-1,1)$ and $(1,5 / 2,-1 / 2)$ respectively with the relative phase $\eta=-\pi / 2$. This model can give an excellent description of lepton flavour mixing, including an atmospheric neutrino mixing angle in the second octant, in terms of only two input parameters. We show that the observed baryon asymmetry can be generated for the lightest right-handed neutrino mass $M_{1}=1.176 \times 10^{11} \mathrm{GeV}$ in SM and $M_{1}=3.992 \times 10^{10} \mathrm{GeV}$ in MSSM with $\tan \beta=5$. We construct an explicit Littlest Seesaw model based on the flavour symmetry $S_{4} \times Z_{5} \times Z_{8}$ in which the desired alignments and the phase $\eta=-\pi / 2$ are achieved.
\end{abstract}

\footnotetext{
${ }^{*}$ E-mail: chenpt@mail.ustc.edu.cn

${ }^{\dagger}$ E-mail: dinggj@ustc.edu.cn

${ }^{\ddagger}$ E-mail: king@soton.ac.uk

${ }^{\S}$ E-mail: 1cc0915@mail.ustc.edu.cn
} 


\section{Introduction}

The Standard Model (SM) has been well established by the discovery of the Higgs boson. However, the discovery of neutrino oscillations implies that neutrinos have masses and there are flavour mixing in lepton sector. Non-zero neutrino masses open up a window to the new physics beyond SM. However, the origin of neutrino mass generation and the flavour mixings in quark and lepton sectors are still unknown [1,2]. In order to elegantly generate the tiny neutrino mass, the most appealing theory seems to be type I seesaw mechanism involving heavy right-handed Majorana neutrinos [3-5].

The type I seesaw mechanism can qualitatively explain the smallness of neutrino masses through the heavy right-handed neutrinos. However, if one doesn't make other assumptions, the seesaw model with three right-handed neutrinos (RHN) contains too many parameters to make any particular predictions for neutrino mass and mixing. As we know, the idea of sequential dominance (SD) $[6,7]$ of right-handed neutrinos is an effective method to produce the mass hierarchy between the two mass squared differences $\Delta m_{21}^{2}$ and $\Delta m_{31}^{2}$ [8], it requires that the mass spectrum of heavy Majorana neutrinos is strongly hierarchical, i.e. $M_{\text {atm }} \ll M_{\text {sol }} \ll M_{\text {dec }}$. It arises from the proposal that a dominant heavy right-handed $(\mathrm{RH})$ neutrino is mainly responsible for the atmospheric neutrino mass, a heavier subdominant $\mathrm{RH}$ neutrino for the solar neutrino mass, and a possible third largely decoupled RH neutrino for the lightest neutrino mass. It leads to an effective two right-handed neutrino (2RHN) model $[9,10]$. This simple idea leads to equally simple predictions which makes the scheme falsifiable. Indeed, the litmus test of such SD is a very light (or massless) neutrino. These predictions will be tested soon. In order to further increase predictive power of the minimal seesaw mechanism, various proposals have been suggested, such as postulating one [11] or two [10] texture zeros in the neutrino Yukawa coupling. The models with two texture zero are excluded by the present data for normal ordering neutrino masses [12-14].

A very predictive minimal seesaw model with one texture zero is the so-called $\operatorname{CSD}(n)$ model [15-24], where the parameter $n$ was usually assumed to be a positive integer. The $\operatorname{CSD}(n) \operatorname{scheme}$ assumes that the two columns of the Dirac neutrino mass matrix are proportional to $(0,1,-1)$ and $(1, n, 2-n)$ respectively in the RHN diagonal basis. As a consequence, the lepton mixing matrix is predicted to be TM1 pattern, the neutrino masses are normal ordering and the lightest neutrino is massless with $m_{1}=0$. At present only the $\operatorname{CSD}(3)$ (also called Littlest Seesaw model) [17-21] and CSD(4) models [22,23] can give rise to phenomenologically viable predictions for lepton mixing parameters and the two neutrino mass squared differences $\Delta m_{21}^{2}$ and $\Delta m_{31}^{2}$.

It has been shown that $\operatorname{CSD}(n)$ can be enforced by a residual symmetry of $S_{4}$ [19] in the semidirect approach where different residual flavour symmetries $G_{l}=Z_{3}^{T}$ and $G_{\nu}=Z_{2}^{S U}$ are assumed in the charged lepton and neutrino sectors. However, it was not possible to identify any residual CP symmetry for $\operatorname{CSD}(n)$ in the semi-direct approach. This means that the parameter $n$ of $\operatorname{CSD}(n)$, which is usually assumed to be integer valued, could in fact be a complex number in general. In order to preserve the predictions of $\operatorname{CSD}(n)$, we would like to fix the parameter $n$ to be real (although not necessarily an integer). This suggests that we should seek to somehow use residual CP symmetry, even though it is not possible within the semi-direct approach.

In the past years, discrete flavour symmetry has been combined with generalized CP symmetry to provide a powerful framework to explain the lepton mixing angles and predict leptonic CP violation phases [25-57]. Furthermore, a simultaneous description of quark and lepton flavour mixing and $\mathrm{CP}$ violation can be achieved through spontaneous breaking of a discrete family symmetry and CP symmetry [52-54]. Since the generalized CP symmetry may play a critical role in understanding the flavour puzzle of SM, recently we extended the widely studied direct model of discrete flavour symmetry [1] to propose a new predictive neutrino mass model building scheme for the minimal seesaw model with two right-handed neutrinos called the tri-direct $\mathrm{CP}$ approach $[58,59]$.

The basic idea of the tri-direct CP approach is that the Yukawa interactions associated with 
each of the two right-handed neutrinos preserve different residual flavour and CP symmetries, and the charged lepton sector also has a different residual flavour symmetry. As a consequence, the flavour and generalized CP symmetry $G_{f} \rtimes H_{C P}$ is spontaneously broken down to $G_{l}, G_{\text {atm }} \rtimes H_{C P}^{\text {atm }}$ and $G_{\text {sol }} \rtimes H_{C P}^{\text {sol }}$ in the charged lepton, "atmospheric" and "solar" right-handed neutrino sectors, respectively [58]. Here $G_{l}$ is an abelian subgroup of $G_{f}$ and it allows the distinction of three generations of charged leptons as usual direct model. The residual subgroups $G_{\text {atm }} \rtimes H_{C P}^{\text {atm }}$ and $G_{\text {sol }} \rtimes H_{C P}^{\text {sol }}$ fix the alignments associated with each right-handed neutrino. We have performed a comprehensive analysis of lepton mixing patterns which can be obtained from the flavour group $S_{4}$ and CP symmetry in the tri-direct CP approach in a model independent fashion [59]. The model construction along the tri-direct $\mathrm{CP}$ approach was also illustrated $[58,59]$. In the minimal seesaw model, a phenomenologically viable pattern of lepton mixing and neutrino masses can also be obtained from the breaking of $A_{5}$ flavour symmetry into three different subgroups in the charged lepton, atmospheric neutrino and solar neutrino sectors [60].

It is remarkable that the original Littlest Seesaw model for $\operatorname{CSD}(3)$ can be reproduced from the tri-direct CP approach [58,59], if the $S_{4}$ flavour symmetry and CP symmetry are broken to the remnant symmetries $Z_{3}^{T}, Z_{2}^{U} \times H_{C P}^{\text {atm }}$ and $Z_{2}^{S U} \times H_{C P}^{\text {sol }}$ in the charged lepton sector, the atmospheric sector and the solar neutrino sector, respectively, corresponding to the $\mathcal{N}_{1}$ case. In this case, one row of the neutrino Dirac mass matrix is proportional to $(0,-1,1)$ and the other row is proportional to $(1,2-x, x)$, where $x$ is enforced to be a real parameter by the residual symmetry, thereby overcoming the previous problem where it could be complex in general. Then the light neutrino mass matrix is determined to be ${ }^{1}[59]$

$$
m_{\nu}=m_{a}\left(\begin{array}{ccc}
0 & 0 & 0 \\
0 & 1 & -1 \\
0 & -1 & 1
\end{array}\right)+m_{s} e^{i \eta}\left(\begin{array}{ccc}
1 & 2-x & x \\
2-x & (x-2)^{2} & (2-x) x \\
x & (2-x) x & x^{2}
\end{array}\right)
$$

where an overall phase has been neglected, $m_{a}, m_{s}, \eta$ and $x$ are four real free parameters. In a concrete model, the parameters $x$ and $\eta$ could be fixed to certain values through the technique of vacuum alignment $[58,59]$. For example, $\operatorname{CSD}(3)$ corresponding to $x=3$ and $\eta=2 \pi / 3$, can be achieved within the $\mathcal{N}_{1}$ case. Then all three mixing angles, two CP phases and three neutrino masses only depend on two real parameters $m_{a}$ and $m_{s}$ which can be determined by the mass squared differences $\Delta m_{21}^{2} \equiv m_{2}^{2}-m_{1}^{2}$ and $\Delta m_{31}^{2} \equiv m_{3}^{2}-m_{1}^{2}$ precisely measured in neutrino oscillation experiments. Then one can extract the predictions for all other mixing parameters. Obviously this kind of model is highly predictive.

In this paper, we shall focus on a particularly interesting example of the $\mathcal{N}_{1}$ case with $x=-1 / 2$ and $\eta=-\pi / 2$, henceforth referred to as the new Littlest Seesaw, which was one of the best fit points found in [59] where the lepton mixing parameters and neutrino masses are predicted to lie in rather narrow regions, with an atmospheric angle in the second octant as preferred by the latest global fits. Motivated by the excellent agreement of this case with experimental data, in this work we develop further this new Littlest Seesaw model in two different ways: we discuss leptogenesis and we also construct a concrete model to demonstrate how it could arise from a realistic theory. We emphasise that the model involves a particularly simple and "maximal" phase $\eta=-\pi / 2$ which is the unique source of $\mathrm{CP}$ violation for both neutrino oscillations and leptogenesis. It is noteworthy that the observed value of the baryon asymmetry $Y_{B}$ of our Universe will be obtained through flavoured thermal leptogenesis in both the SM and the Minimal Supersymmetric Standard Model (MSSM). We will propose an explicit supersymmetric (SUSY) model in the framework of minimal seesaw mechanism with $2 \mathrm{RHN}$ based on $S_{4} \rtimes H_{C P}$ and show that the mass hierarchies of the

\footnotetext{
${ }^{1}$ Note that the seesaw mechanism results in a light effective Majorana mass matrix was defined in the convention $\mathcal{L}_{\text {eff }}=-\frac{1}{2} \overline{\nu_{L}^{c}} m_{\nu} \nu_{L}+$ h.c. Also note that here the second entries of the vacuum alignments which enter the Dirac mass matrix are multiplied by minus one as compared to the usual Littlest Seesaw convention.
} 
charged lepton and the light neutrino mass matrix in Eq. (1) with $x=-1 / 2$ and $\eta=-\pi / 2$ may be naturally derived in such a model.

The rest of this paper is organized as follows. In section 2 , we revisit the $\mathcal{N}_{1}$ case of tri-direct CP models with the alignments $\left\langle\phi_{\mathrm{atm}}\right\rangle \propto(0,1,-1)^{T},\left\langle\phi_{\text {sol }}\right\rangle \propto(1, x, 2-x)^{T}$ which can be derived from the $S_{4}$ flavour symmetry in combination with CP symmetry, assuming the $\mathcal{N}_{1}$ residual symmetry. We show that the new Littlest Seesaw model, which corresponds to a benchmark point in the $\mathcal{N}_{1}$ case with $x=-1 / 2$ and $\eta=-\pi / 2$, provides an excellent fit to the experimental data of lepton mixing angles and neutrino masses. We study the predictions of the new Littlest Seesaw model for leptogenesis in the section 3, and show that the observed baryon asymmetry of the Universe can be produced for certain values of the lightest right-handed neutrino mass. In section 4 , we construct a supersymmetric littlest tri-direct $\mathrm{CP}$ model based on the flavour symmetry $S_{4} \times Z_{5} \times Z_{8}$, the alignment parameter $x=-1 / 2$ and relative phase $\eta=-\pi / 2$ are achieved. The predictions for the charged lepton flavour violation radiative decays $l_{i} \rightarrow l_{j} \gamma$ are studied, and we show a UV completion of the model. In section 5, we summarize our main results and draw the conclusions. We present the group theory and the Clebsch-Gordan coefficients of the $S_{4}$ group in Appendix A.

\section{The $\mathcal{N}_{1}$ case of tri-direct $\mathrm{CP}$ models revisited}

The tri-direct $\mathrm{CP}$ approach is based on the minimal seesaw model with $2 \mathrm{RHN}$. We denote the two right-handed neutrinos as $N_{\text {atm }}^{c}$ (called "atmospheric") and $N_{\text {sol }}^{c}$ (called "solar"). Then the most general Lagrangian of the minimal seesaw model can be written as

$$
\mathcal{L}=-y_{l} L \phi_{l} E^{c}-y_{\mathrm{atm}} L \phi_{\mathrm{atm}} N_{\mathrm{atm}}^{c}-y_{\mathrm{sol}} L \phi_{\mathrm{sol}} N_{\mathrm{sol}}^{c}-\frac{1}{2} x_{\mathrm{atm}} \xi_{\mathrm{atm}} N_{\mathrm{atm}}^{c} N_{\mathrm{atm}}^{c}-\frac{1}{2} x_{\mathrm{sol}} \xi_{\mathrm{sol}} N_{\mathrm{sol}}^{c} N_{\mathrm{sol}}^{c}+\text { h.c. },
$$

where two-component fermion notation for the fermion fields is adopted. The lepton doublets $L$ are assumed to transform as an irreducible triplet under $S_{4}(L \sim \mathbf{3}), \phi_{\text {atm }}$ and $\phi_{\text {sol }}$ can be either Higgs fields or combinations of the electroweak Higgs doublet together with flavons, and they are also $S_{4}$ triplets $\left(\phi_{\mathrm{atm}} \sim \mathbf{3}\right.$ and $\left.\phi_{\text {sol }} \sim \mathbf{3}^{\prime}\right)$. The two right-handed neutrinos are singlets of $S_{4}$ with $N_{\mathrm{atm}}^{c} \sim \mathbf{1}$ and $N_{\mathrm{sol}}^{c} \sim \mathbf{1}^{\prime}$, the two flavons $\xi_{\mathrm{atm}}$ and $\xi_{\text {sol }}$ are invariant under $S_{4}$. The combination of flavons $\phi_{l}$ and the right-handed charged leptons $E^{c} \equiv\left(e^{c}, \mu^{c}, \tau^{c}\right)^{T}$ must be embedded into the faithful three-dimensional representation 3 of $S_{4}$. Moreover, all coupling constants $y_{\mathrm{atm}}, y_{\mathrm{sol}}, x_{\mathrm{atm}}$ and $x_{\text {sol }}$ are real because of the generalized CP symmetry.

We have performed an exhaustive analysis of all possible residual symmetries arising from $S_{4} \rtimes H_{C P}$ in tri-direct $\mathrm{CP}$ approach and the resulting predictions for neutrino masses and flavour mixing parameters in [59]. Many independent phenomenologically viable residual symmetry cases are found (eight cases for normal ordering and eighteen cases for inverted ordering). In the present work, we shall consider the breaking pattern in which the residual symmetries in the charged lepton, atmospheric neutrino and solar neutrino sectors are $Z_{3}^{T}, Z_{2}^{U} \times H_{C P}^{\text {atm }}$ and $Z_{2}^{S U} \times H_{C P}^{\text {sol }}$ respectively, the two residual CP symmetries are $H_{C P}^{\text {atm }}=\{1, U\}$ and $H_{C P}^{\text {sol }}=\{1, S U\}$. This is exactly the case $\mathcal{N}_{1}$ of Ref. [59]. The residual symmetries in atmospheric neutrino and solar neutrino sectors require that the vacuum expectation values $(\mathrm{VEVs})$ of the flavons $\phi_{\mathrm{atm}}$ and $\phi_{\text {sol }}$ should take the following form

$$
\left\langle\phi_{\text {atm }}\right\rangle=v_{\text {atm }}(0,1,-1)^{T}, \quad\left\langle\phi_{\text {sol }}\right\rangle=v_{\text {sol }}(1, x, 2-x)^{T},
$$

where the parameters $v_{\text {atm }}, v_{\text {sol }}$ and $x$ are real. Applying the well-known seesaw formula, the light neutrino mass matrix $m_{\nu}$ is really given by Eq. (1).

In our working basis (see Appendix A), requiring that the subgroup $Z_{3}^{T}$ is a symmetry of the charged neutrino mass matrix $m_{l}$ entails that $m_{l}^{\dagger} m_{l}$ is diagonal and thus does not contribute to the 
lepton mixing. The lepton mixing matrix is found to be of the following form [59]:

$$
U_{P M N S}=\left(\begin{array}{ccc}
\sqrt{\frac{2}{3}} & \frac{\cos \theta}{\sqrt{3}} & \frac{e^{i \psi} \sin \theta}{\sqrt{3}} \\
-\frac{1}{\sqrt{6}} & \frac{\cos \theta}{\sqrt{3}}+\frac{e^{-i \psi} \sin \theta}{\sqrt{2}} & \frac{e^{i \psi} \sin \theta}{\sqrt{3}}-\frac{\cos \theta}{\sqrt{2}} \\
-\frac{1}{\sqrt{6}} & \frac{\cos \theta}{\sqrt{3}}-\frac{e^{-i \psi} \sin \theta}{\sqrt{2}} & \frac{\cos \theta}{\sqrt{2}}+\frac{e^{i \psi} \sin \theta}{\sqrt{3}}
\end{array}\right) P_{\nu},
$$

where $P_{\nu}=\operatorname{diag}\left(1, e^{i(\psi+\rho) / 2}, e^{i(-\psi+\sigma) / 2}\right)$ is a diagonal phase matrix. We see that the first column of the mixing matrix is in common with that of the tri-bimaximal mixing matrix, and the so-called TM1 mixing matrix is obtained. The neutrino mass spectrum is normal ordering, the lightest neutrino is massless $\left(m_{1}=0\right)$ since only two right-handed neutrinos are involved. The other two non-zero light neutrino masses $m_{2}$ and $m_{3}$ are given by

$$
m_{2}^{2}=\frac{m_{a}^{2}}{2}\left[9 r^{2}+w^{2}+12 r^{2}(x-1)^{2}-\sqrt{B}\right], \quad m_{3}^{2}=\frac{m_{a}^{2}}{2}\left[9 r^{2}+w^{2}+12 r^{2}(x-1)^{2}+\sqrt{B}\right],
$$

where

$$
\begin{aligned}
& r=m_{s} / m_{a}, \quad w=2 \sqrt{1+r^{2}(x-1)^{4}+2 r(x-1)^{2} \cos \eta}, \\
& B=\left(9 r^{2}-w^{2}\right)^{2}+24 r^{2}(x-1)^{2} A, \\
& A=9 r^{2}+w^{2}+6 r w \cos \left(\eta-\phi_{w}\right), \quad \phi_{w}=\arg \left(1+r(x-1)^{2} e^{i \eta}\right) .
\end{aligned}
$$

The expressions for the angles and phases $\theta, \psi, \rho$ and $\sigma$ in Eq. (4) are:

$$
\begin{aligned}
& \cos 2 \theta=\frac{w^{2}-9 r^{2}}{\sqrt{B}}, \quad \sin 2 \theta=\frac{2 \sqrt{6 A} r(x-1)}{\sqrt{B}}, \quad \sin \psi=-\frac{w \sin \left(\eta-\phi_{w}\right)}{\sqrt{A}}, \\
& \cos \psi=\frac{3 r+w \cos \left(\eta-\phi_{w}\right)}{\sqrt{A}}, \quad \sin (\rho-\sigma)=\frac{3 r w m_{a}^{2} \sqrt{B} \sin \left(\eta-\phi_{w}\right)}{m_{2} m_{3} A} .
\end{aligned}
$$

From the lepton mixing matrix in Eq. (4), one can straightforwardly extract the following results for the lepton mixing angles and $\mathrm{CP}$ invariants,

$$
\begin{aligned}
& \sin ^{2} \theta_{13}=\frac{\sin ^{2} \theta}{3}=\frac{1}{6}\left(1-\frac{w^{2}-9 r^{2}}{\sqrt{B}}\right), \quad \sin ^{2} \theta_{12}=\frac{2 \cos ^{2} \theta}{5+\cos 2 \theta}=\frac{1}{3}\left(1-2 \tan ^{2} \theta_{13}\right), \\
& \sin ^{2} \theta_{23}=\frac{1}{2}-\frac{\sqrt{6} \sin 2 \theta \cos \psi}{5+\cos 2 \theta}=\frac{1}{2}-\frac{12 r(x-1)\left[3 r+w \cos \left(\eta-\phi_{w}\right)\right]}{5 \sqrt{B}+w^{2}-9 r^{2}}, \\
& J_{C P}=\frac{\sin 2 \theta \sin \psi}{6 \sqrt{6}}=-\frac{w r(x-1) \sin \left(\eta-\phi_{w}\right)}{3 \sqrt{B}}, \\
& I_{1}=\frac{1}{36} \sin ^{2} 2 \theta \sin (\rho-\sigma)=\frac{2 r^{3} w(x-1)^{2} \sin \left(\eta-\phi_{w}\right)}{m_{2} m_{3} \sqrt{B}},
\end{aligned}
$$

where $J_{C P}$ is the Jarlskog invariant [61] and $I_{1}$ is the Majorana invariant [62] related to the Majorana phase $\varphi$. We find that all mixing parameters and mass ratio $m_{2} / m_{3}$ depend on the three input parameters $x, \eta$ and $r=m_{s} / m_{a}$. However, the neutrino absolute masses $m_{2}$ and $m_{3}$ depend on all the four input parameters $x, \eta, m_{a}$ and $r$. We find that the agreement with data is optimised by choosing

$$
m_{a}=23.133 \mathrm{meV}, \quad r=0.135, \quad \eta=-0.542 \pi, \quad x=-0.615,
$$

which give rise to the following values of observables

$$
\begin{aligned}
& \sin ^{2} \theta_{13}=0.02241, \quad \sin ^{2} \theta_{12}=0.318, \quad \sin ^{2} \theta_{23}=0.582, \quad \delta_{C P}=-0.382 \pi, \quad \varphi=0.333 \pi, \\
& m_{1}=0 \mathrm{meV}, \quad m_{2}=8.597 \mathrm{meV}, \quad m_{3}=50.249 \mathrm{meV}, \quad m_{e e}=3.112 \mathrm{meV},
\end{aligned}
$$




\begin{tabular}{|l|c|c|c|c|c|c|c|c|c|c|c|c|c|}
\hline \hline$\left\langle\phi_{\text {sol }}\right\rangle / v_{\phi_{s}}$ & $x$ & $\eta$ & $m_{a}(\mathrm{meV})$ & $r$ & $\chi_{\min }^{2}$ & $\sin ^{2} \theta_{13}$ & $\sin ^{2} \theta_{12}$ & $\sin ^{2} \theta_{23}$ & $\delta_{C P} / \pi$ & $\varphi / \pi$ & $m_{2}(\mathrm{meV})$ & $m_{3}(\mathrm{meV})$ & $m_{e e}(\mathrm{meV})$ \\
\hline$(1,3,-1)^{T}$ & 3 & $\pm \frac{2 \pi}{3}$ & 26.850 & 0.0997 & 24.861 & 0.0221 & 0.318 & 0.488 & $\mp 0.516$ & $\mp 0.403$ & 8.579 & 50.272 & 2.677 \\
\hline$(1,-1,3)^{T}$ & -1 & $\pm \frac{2 \pi}{3}$ & 26.796 & 0.101 & 13.744 & 0.0225 & 0.318 & 0.513 & \pm 0.482 & $\mp 0.401$ & 8.632 & 50.210 & 2.696 \\
\hline$(1,4,-2)^{T}$ & 4 & $\pm \frac{4 \pi}{5}$ & 35.249 & 0.0564 & 14.358 & 0.0241 & 0.317 & 0.575 & $\mp 0.398$ & $\mp 0.474$ & 8.315 & 50.610 & 1.990 \\
\hline$\left(1, \frac{7}{2},-\frac{3}{2}\right)^{T}$ & \multirow{2}{*}{$\frac{7}{2}$} & $\pm \frac{3 \pi}{4}$ & 31.123 & 0.0734 & 7.823 & 0.0231 & 0.318 & 0.541 & $\mp 0.444$ & $\mp 0.447$ & 8.459 & 50.429 & 2.284 \\
\hline$\left(1, \frac{10}{3},-\frac{4}{3}\right)^{T}$ & $\frac{10}{3}$ & \pm 3.016 & 0.0673 & 9.143 & 0.0209 & 0.319 & 0.589 & $\mp 0.366$ & $\mp 0.544$ & 8.802 & 50.014 & 2.222 \\
\hline$\left(1,-\frac{1}{2}, \frac{5}{2}\right)^{T}$ & $-\frac{1}{2}$ & $\pm \frac{\pi}{2}$ & 22.366 & 0.145 & 2.487 & 0.0220 & 0.318 & 0.599 & \pm 0.354 & $\mp 0.317$ & 8.670 & 50.167 & 3.241 \\
\hline$\left(1,-\frac{2}{3}, \frac{8}{3}\right)^{T}$ & $-\frac{2}{3} \pm \frac{3 \pi}{5}$ & 24.571 & 0.122 & 14.594 & 0.0212 & 0.319 & 0.545 & \pm 0.435 & $\mp 0.383$ & 8.889 & 49.911 & 3.009 \\
\hline$\left(1,-\frac{3}{4}, \frac{11}{4}\right)^{T}$ & $-\frac{3}{4} \pm \frac{3 \pi}{5}$ & 24.579 & 0.120 & 3.600 & 0.0222 & 0.318 & 0.551 & \pm 0.429 & $\mp 0.367$ & 8.670 & 50.167 & 2.949 \\
\hline$\left(1,-\frac{3}{5}, \frac{13}{5}\right)^{T}$ & $-\frac{3}{5}$ & $\pm \frac{\pi}{2}$ & 22.220 & 0.142 & 11.666 & 0.0232 & 0.318 & 0.606 & \pm 0.347 & $\mp 0.297$ & 8.309 & 50.618 & 3.155 \\
\hline$\left(1,-\frac{4}{5}, \frac{14}{5}\right)^{T}$ & $-\frac{4}{5} \pm \frac{3 \pi}{5}$ & 24.585 & 0.118 & 3.249 & 0.0228 & 0.318 & 0.554 & \pm 0.425 & $\mp 0.357$ & 8.534 & 50.333 & 2.912 \\
\hline$\left(1,-\frac{5}{6}, \frac{17}{6}\right)^{T}$ & $-\frac{5}{6}$ & $\pm \frac{3 \pi}{5}$ & 24.590 & 0.117 & 5.588 & 0.0231 & 0.318 & 0.556 & \pm 0.422 & $\mp 0.350$ & 8.443 & 50.451 & 2.887 \\
\hline \hline
\end{tabular}

Table 1: Some benchmark values of the parameters $x$ and $\eta$ and the corresponding predictions for the lepton mixing angles, CP violation phases, neutrino masses and the effective Majorana mass $m_{e e}$. These results are benchmark examples in the $\mathcal{N}_{1}$ class of tri-direct CP models [59]. Notice that the lightest neutrino mass is vanishing $m_{1}=0$.

where $m_{e e}$ refers to the effective Majorana mass in neutrinoless double beta decay, and $\varphi$ is the Majorana phase. These predictions for lepton mixing angles agree with the experimental data quite well, and the global minimum of the $\chi^{2}$ function is $\chi_{\min }^{2}=0.384$. Note that the $\chi^{2}$ function includes the contributions of three mixing angles and two squared mass differences as usual. Because the indication of a preferred value of the Dirac phase $\delta_{C P}$ from global data analyses is rather weak [8], we do not include any information on $\delta_{C P}$ in the $\chi^{2}$ function. We emphasise that the values of the parameter $x, \eta, r$ and $m_{a}$ are not fixed by the residual symmetry, and can only be fixed by explicit model construction. This task is easier for the simpler values of $x$ and $\eta$ where the solar vacuum alignment $\left\langle\phi_{\text {sol }}\right\rangle$ is easier to achieve, therefore we are interested in the simplest values of these parameters.

We report the results of $\chi^{2}$ analysis for some representative values of $x$ and $\eta$ in table 1 . Once the values of $x$ and $\eta$ are fixed, all the mixing parameters and neutrino masses only depend on the input parameters $m_{a}$ and $r$ whose values can be determined by the mass squared differences $\Delta m_{21}^{2}$ and $\Delta m_{31}^{2}$. Then the three lepton mixing angles, two $\mathrm{CP}$ violation phases and the absolute neutrino mass scale are uniquely predicted by the theory. We notice that the effective Majorana mass $m_{e e}$ lies in the range of 1 to $4 \mathrm{meV}$, consequently it is impossible to be measured in foreseeable future.

The original Littlest Seesaw model [18-21] corresponds to the cases of $(x, \eta)=(3,2 \pi / 3)$, $(-1,-2 \pi / 3)$, and the $\operatorname{CSD}(4)$ model $[22,23]$ can be exactly reproduced for $(x, \eta)=(4,4 \pi / 5)$. From table 1 , we see that the values $(x, \eta)=(-1 / 2, \pm \pi / 2),(-3 / 4, \pm 3 \pi / 5)$ and $(-4 / 5, \pm 3 \pi / 5)$ can give rise to a smaller $\chi_{\min }^{2}$ than the original Littlest Seesaw model and CSD(4) model [18-23]. We have shown $\chi_{\min }^{2}$ as a function of $\eta$ for $x=3,4,-1 / 2,-3 / 4,-3 / 5$ in figure 1 . Moreover, we plot the contour regions for the $3 \sigma$ intervals of mixing angles $\theta_{13}$ and $\theta_{23}$ and mass ratio $m_{2} / m_{3}$ in the plane $r$ versus $\eta / \pi$ in figure 2. The result for $\theta_{12}$ is not displayed here, because it is related to the reactor angle $\theta_{13}$ by the TM1 mixing sum rule $\cos ^{2} \theta_{12} \cos ^{2} \theta_{13}=2 / 3$ which leads to $0.316 \leq \sin ^{2} \theta_{12} \leq 0.319$ for the $3 \sigma$ allowed range of $\theta_{13}$ [8].

From figures 1 and 2, we notice that the values of $\chi_{\min }^{2}$ is quite sensitive to the phase $\eta$ and predictions for the mixing angles and neutrino masses can agree very well with the experimental data for certain choices of $\eta$. Henceforth we shall focus on the new Littlest Seesaw model defined by the simple values $x=-1 / 2$ and $\eta= \pm \pi / 2$ which give a phenomenologically successful and predictive description of lepton mixing parameters and neutrino masses, as highlighted with cyan 


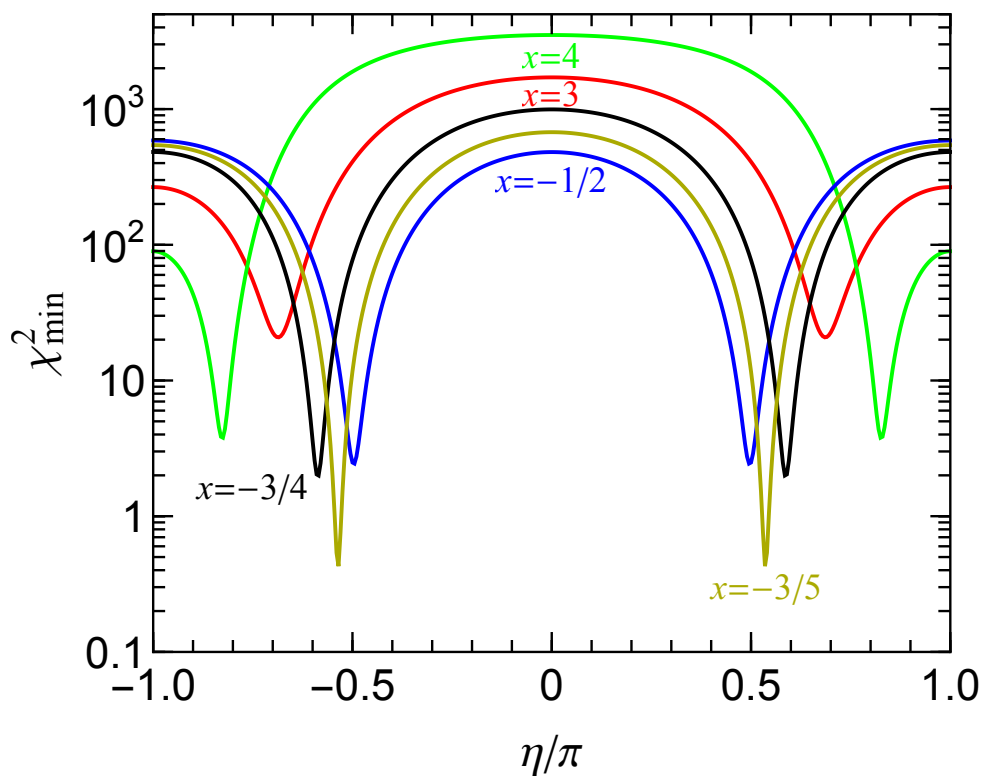

Figure 1: Variation of $\chi^{2}$ with respect to the phase $\eta$ for the typical values of $x=3,4,-1 / 2,-3 / 4,-3 / 5$, for the $\mathcal{N}_{1}$ case of tri-direct CP models.

background in table 1. Moreover, the corresponding vacuum alignment $\left\langle\phi_{\text {sol }}\right\rangle \propto(1,-1 / 2,5 / 2)$ and the phase $\eta= \pm \pi / 2$ should be easy to realize in a concrete model. This new Littlest Seesaw model and the original Littlest Seesaw model differ in their predictions for $\theta_{23}$ and $\delta_{C P}$. The atmospheric mixing angle $\theta_{23}$ deviates from maximal mixing in the new Littlest Seesaw model while it is close to $45^{\circ}$ in the original Littlest Seesaw. Since deviation of $\theta_{23}$ from maximal mixing is preferred by the present data [8], the new littlest tri-direct $\mathrm{CP}$ model provides a better fit to the data of $\theta_{23}$ than the original Littlest Seesaw.

\subsection{The new Littlest Seesaw: $x=-1 / 2, \eta=-\pi / 2$}

Before getting into too many technicalities of model construction, we analyze the predictions for lepton mixing parameters and neutrino masses for $x=-1 / 2, \eta=-\pi / 2$. In this case, the light neutrino mass matrix in Eq. (1) becomes

$$
m_{\nu}=m_{a}\left(\begin{array}{ccc}
0 & 0 & 0 \\
0 & 1 & -1 \\
0 & -1 & 1
\end{array}\right)-\frac{i m_{s}}{4}\left(\begin{array}{ccc}
4 & 10 & -2 \\
10 & 25 & -5 \\
-2 & -5 & 1
\end{array}\right)
$$

We note that all lepton mixing parameters and mass ratio $m_{2} / m_{3}$ are determined by only a single parameter $r=m_{s} / m_{a}$. The expressions for the three lepton mixing angles and the CP invariants are given by

$$
\begin{array}{ll}
\sin ^{2} \theta_{13}=\frac{1}{6}\left(1-\frac{45 r^{2}+16}{C_{r}}\right), & \sin ^{2} \theta_{12}=1-\frac{4 C_{r}}{5 C_{r}+45 r^{2}+16}, \\
\sin ^{2} \theta_{23}=\frac{1}{2}+\frac{540 r^{2}}{5 C_{r}+45 r^{2}+16}, & J_{C P}=-\frac{4 r}{C_{r}},
\end{array}
$$

with

$$
C_{r}=\left.4 \sqrt{B}\right|_{x=-1 / 2, \eta=-\pi / 2}=\sqrt{\left(225 r^{2}+16\right)^{2}-2304 r^{2}} .
$$



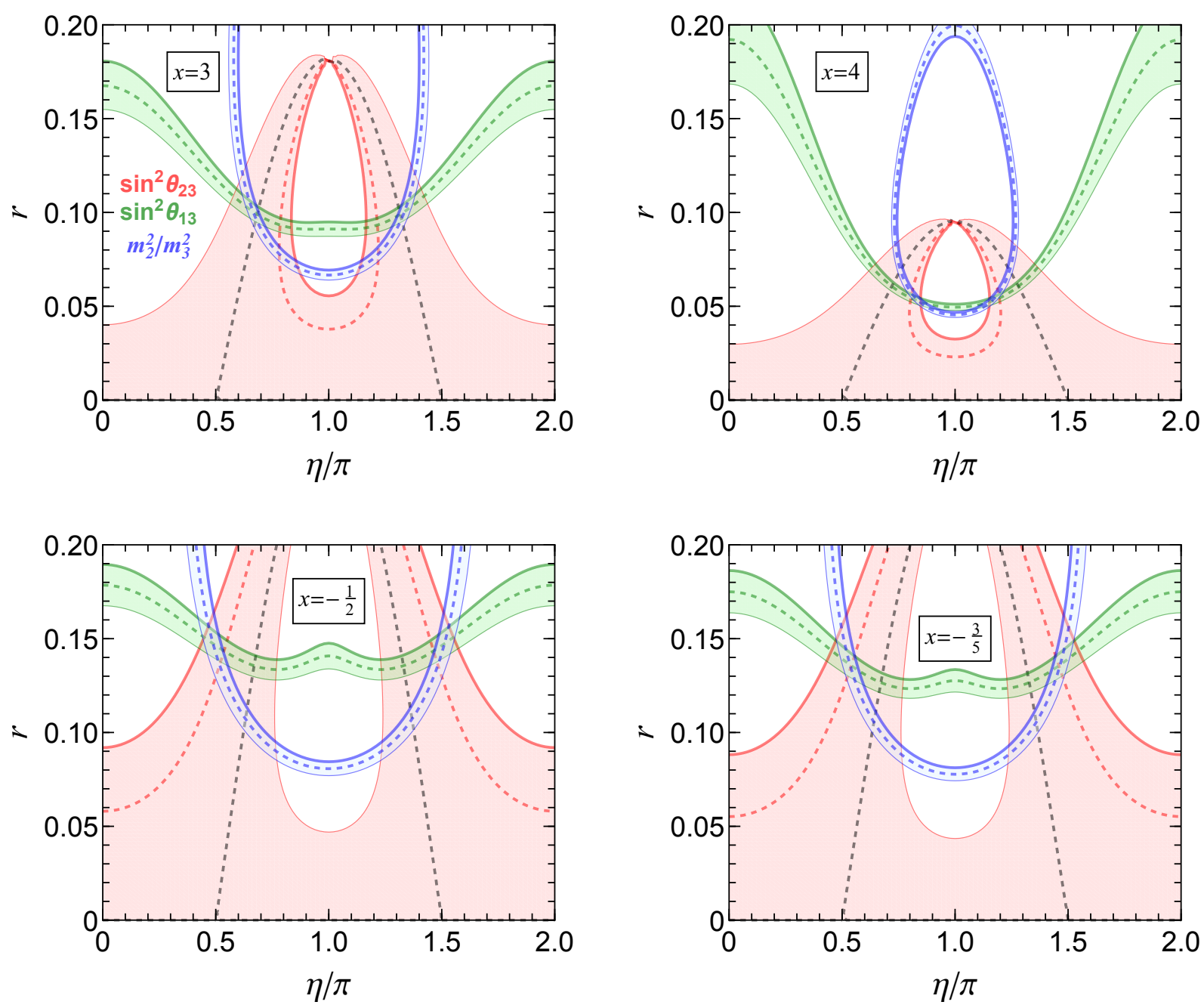

Figure 2: Contour plots of $\sin ^{2} \theta_{13}, \sin ^{2} \theta_{23}$ and $m_{2} / m_{3}$ in the $\eta / \pi-r$ plane for $x=3,4,-1 / 2$ and $-3 / 5$, for the $\mathcal{N}_{1}$ case of tri-direct CP models. The red, green and blue areas denote the $3 \sigma$ contour regions of $\sin ^{2} \theta_{23}, \sin ^{2} \theta_{13}$ and the mass ratio $m_{2}^{2} / m_{3}^{2}$ respectively. The dashed lines denote the best fit values from NuFIT 4.0.

Notice that $\theta_{23}$ is predicted to lie in the second octant, it is preferred by the present neutrino oscillation data [8]. As both $\theta_{13}$ and $\theta_{23}$ depend on a single parameter $r$, a sum rule between them can be obtained ${ }^{2}$

$$
\sin ^{2} \theta_{23}=\frac{1+4 \sin ^{2} \theta_{13}+\sqrt{1+28 \sin ^{2} \theta_{13}\left(1-3 \sin ^{2} \theta_{13}\right)}}{4 \cos ^{2} \theta_{13}} .
$$

The two non-zero neutrino masses can be read off from Eq. (5) as,

$$
m_{2}^{2}=\frac{1}{8} m_{a}^{2}\left(16+225 r^{2}-C_{r}\right), \quad m_{3}^{2}=\frac{1}{8} m_{a}^{2}\left(16+225 r^{2}+C_{r}\right) .
$$

It is easy to see that the mass ratio $m_{2} / m_{3}$ only depends on the parameter $r$. Consequently we can express the mass ratio $m_{2}^{2} / m_{3}^{2}$ in terms of $\theta_{13}$ as

$$
\frac{m_{2}^{2}}{m_{3}^{2}}=\frac{10 \sin ^{2} \theta_{13}\left(3 \sin ^{2} \theta_{13}-1\right)+\sqrt{1+28 \sin ^{2} \theta_{13}\left(1-3 \sin ^{2} \theta_{13}\right)}-1}{2 \sin ^{2} \theta_{13}\left(15 \sin ^{2} \theta_{13}-8\right)+2} .
$$

We plot the dependence of all lepton mixing parameters and mass ratio $m_{2} / m_{3}$ on the parameter

\footnotetext{
${ }^{2}$ The sum rule for $\theta_{12}$ is $\cos ^{2} \theta_{12} \cos ^{2} \theta_{13}=2 / 3$ which holds true for all TM1 models.
} 

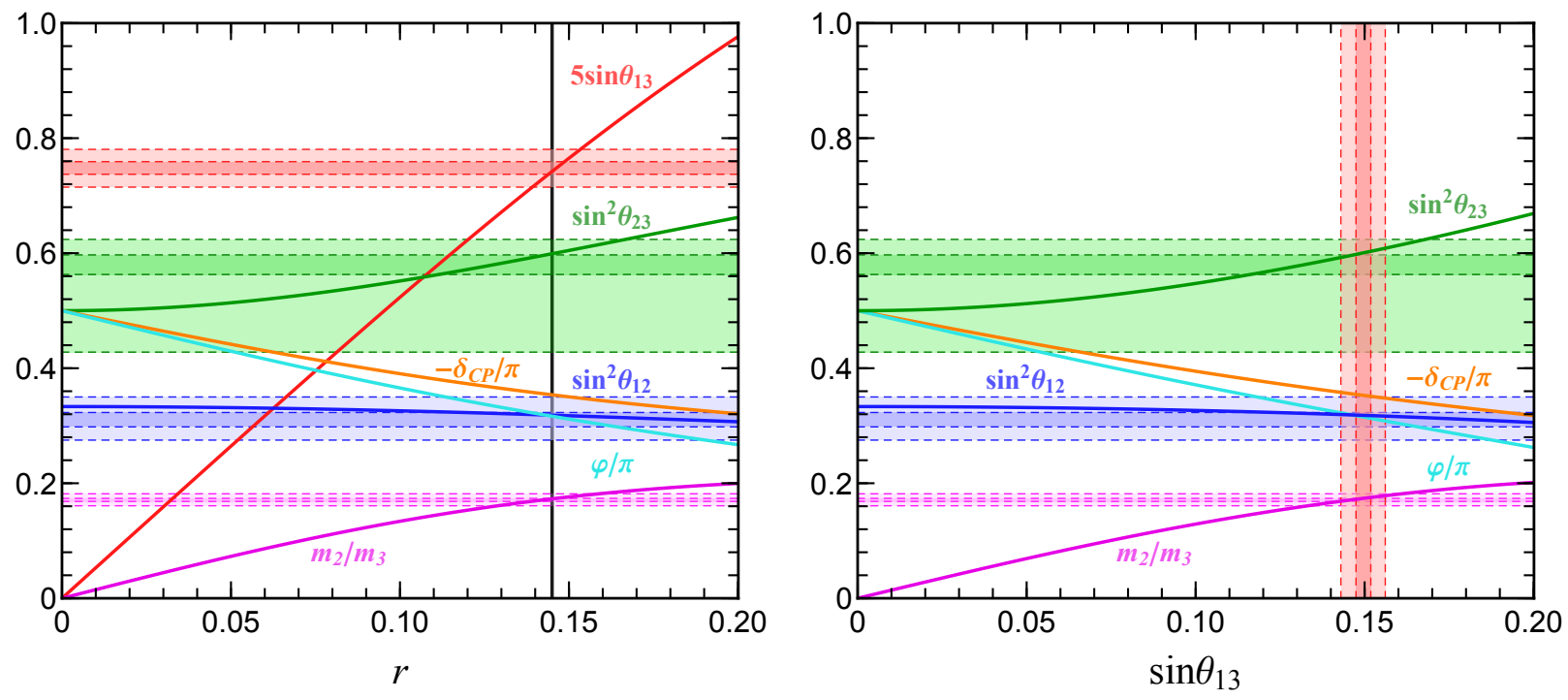

Figure 3: The predictions of the new Littlest Seesaw model with $x=-1 / 2, \eta=-\pi / 2$ for the mixing parameters and mass ratio $m_{2} / m_{3}$. The shaded regions represent the $1 \sigma$ and $3 \sigma$ ranges of each mixing parameter and mass ratio [8]. On the left panel, the values of mixing parameters and mass ratio are predicted with respect to $r$ and the black vertical line denotes the best fit value $r_{\mathrm{bf}}=0.145$. On the right panel, we show the predictions for mixing parameters and mass ratio as functions of $\sin \theta_{13}$.

$r$ in figure 3. Eliminating the input parameter $r$, we can relate all above physical observables to the reactor mixing angle $\theta_{13}$. We see from figure 3 that the three lepton mixing angles and neutrino mass ratio are within their $1 \sigma$ ranges at the best fit point $r=0.145$. The best fitting values of Dirac $\mathrm{CP}$ phase and Majorana CP phase are $\delta_{C P} \simeq-0.354 \pi$ and $\varphi \simeq 0.316 \pi$, respectively. We numerically scan over the parameter space of $m_{a}$ and $r$, and find the viable range of $r$ is $r \in[0.139,0.153]$ to be compatible with the present neutrino oscillation data at $3 \sigma$ level [8]. Furthermore, we find the neutrino masses and mixing parameters are predicted to lie in the following rather narrow regions,

$$
\begin{aligned}
& 0.3167 \leq \sin ^{2} \theta_{12} \leq 0.3194, \quad 0.02044 \leq \sin ^{2} \theta_{13} \leq 0.02437, \quad 0.593 \leq \sin ^{2} \theta_{23} \leq 0.609 \\
& -0.358 \leq \delta_{C P} / \pi \leq-0.348, \quad 0.308 \leq \varphi / \pi \leq 0.322, \quad 3.084 \mathrm{meV} \leq m_{e e} \leq 3.388 \mathrm{meV}, \\
& 8.319 \mathrm{meV} \leq m_{2} \leq 8.950 \mathrm{meV}, \quad 49.305 \mathrm{meV} \leq m_{3} \leq 51.206 \mathrm{meV}
\end{aligned}
$$

Therefore this new Littlest Seesaw model is very predictive and it should be easily excluded by precise measurement of $\theta_{12}, \theta_{23}$ and $\delta_{C P}$ in forthcoming neutrino facilities.

\subsection{The new Littlest Seesaw as a limiting case of three right-handed neutrinos}

We shall extend the idea of Littlest seesaw to the 3RHN model in the following. We denote the $3 \mathrm{RHN}$ as $N_{\mathrm{atm}}^{c}, N_{\mathrm{sol}}^{c}$ and $N_{\mathrm{dec}}^{c}$. Then for the seesaw Lagrangian in Eq. (2), the two additional terms related to the third right-handed neutrino $N_{\mathrm{dec}}^{c}$ can be written as

$$
\Delta \mathcal{L}=-y_{\mathrm{dec}} L \phi_{\mathrm{dec}} N_{\mathrm{dec}}^{c}-\frac{1}{2} x_{\mathrm{dec}} \xi_{\mathrm{dec}} N_{\mathrm{dec}}^{c} N_{\mathrm{dec}}^{c}+\text { h.c. } .
$$

Here the flavon $\phi_{\mathrm{dec}}$ is assigned to transform as $S_{4}$ triplet $\mathbf{3}$, both $\xi_{\mathrm{dec}}$ and $N_{\mathrm{dec}}^{c}$ are invariant under the actions of $S_{4}$. As an example, we consider the case that the residual symmetry in the decoupled neutrino sector is $Z_{2}^{T^{2} S T} \times H_{C P}^{\mathrm{dec}}$ with $H_{C P}^{\mathrm{dec}}=\left\{S U, T S T^{2} U\right\}$. Then the most general VEV of $\phi_{\mathrm{dec}}$ which preserves the above residual symmetry is

$$
\left\langle\phi_{\mathrm{dec}}\right\rangle \propto\left(1, \omega, \omega^{2}\right)^{T} .
$$


Then the neutrino mass matrix in Eq. (11) becomes

$$
m_{\nu}=m_{a}\left(\begin{array}{ccc}
0 & 0 & 0 \\
0 & 1 & -1 \\
0 & -1 & 1
\end{array}\right)-\frac{i}{4} m_{a} r\left(\begin{array}{ccc}
4 & 10 & -2 \\
10 & 25 & -5 \\
-2 & -5 & 1
\end{array}\right)+m_{a} r^{\prime} e^{i \eta^{\prime}}\left(\begin{array}{ccc}
1 & \omega^{2} & \omega \\
\omega^{2} & \omega & 1 \\
\omega & 1 & \omega^{2}
\end{array}\right),
$$

where $m_{a}, r, r^{\prime}, \eta$ and $\eta^{\prime}$ are real. The first two terms coincide with those of the new Littlest Seesaw in Eq. (11), and the last term arises from the third decoupled right-handed neutrinos. An particularly interesting example is the case of $\eta^{\prime}=0$, it predicts the best fit values of the mixing parameters as follows

$$
\begin{aligned}
& m_{a}=22.663 \mathrm{meV}, \quad r=0.141, \quad r^{\prime}=0.00834, \quad \eta=-\pi / 2, \quad \eta^{\prime}=0, \quad \chi_{\min }^{2}=1.157, \\
& \sin ^{2} \theta_{13}=0.0224, \quad \sin ^{2} \theta_{12}=0.318, \quad \sin ^{2} \theta_{23}=0.595, \quad \delta_{C P}=-0.363 \pi, \quad \alpha_{21}=0.394 \pi, \\
& \alpha_{31}=0.0716 \pi, \quad m_{1}=0.285 \mathrm{meV}, \quad m_{2}=8.577 \mathrm{meV}, \quad m_{3}=50.283, \quad m_{e e}=3.197 \mathrm{meV} .
\end{aligned}
$$

We see $r^{\prime} \ll r \ll 1$ such that the condition of constrained sequence dominance is well satisfied. Therefore our new Littlest Seesaw with $2 \mathrm{RHN}$ can be regarded as a decoupling limit of the 3RHN model in the case of $M_{\mathrm{dec}} \gg M_{\mathrm{atm}}, M_{\mathrm{sol}}$. Comparing the best fit values of 3RHN model in Eq. (20) with those of $2 \mathrm{RHN}$ model with $x=-1 / 2$ and $\eta=-\pi / 2$ in table 1 , we find that the $2 \mathrm{RHN}$ model is a good approximation of the $3 \mathrm{RHN}$ model.

\section{Predictions for leptogenesis in the new Littlest Seesaw model}

It is well-known fact that there is a predominance of matter over antimatter present in the observable Universe. The value of baryon asymmetry of the Universe normalised to the entropy density is [63],

$$
Y_{B}=(0.870300 \pm 0.011288) \times 10^{-10} \quad(95 \% \mathrm{CL}) .
$$

Apart from elegantly explaining the tiny neutrino masses, the seesaw mechanism provides a simple and attractive mechanism for understanding the matter-antimatter asymmetry of the Universe via leptogenesis [64]. The out-of-equilibrium decays of right-handed neutrinos in the early Universe generates a lepton asymmetry because of the CP violating Yukawa couplings. The lepton asymmetry is subsequently converted into a baryon asymmetry via sphaleron processes in the SM.

In our concerned model, the phase $\eta$ is the unique source of $\mathrm{CP}$ violation, and it controls $\mathrm{CP}$ violation in both neutrino oscillations and leptogenesis. Therefore the measurable CP violation in future neutrino oscillation experiments are closely related to the baryon asymmetry of the Universe. In the present work, we shall focus on the simplest version of the leptogenesis in which the lepton asymmetry is dominantly generated by the interactions and decay of the lightest right-handed neutrino. The phase $\eta$ is fixed to $\eta=-\pi / 2$ in the new Littlest Seesaw model, and it yields a Dirac $\mathrm{CP}$ violation phase $\delta_{C P} \simeq 1.646 \pi$. In this section, we shall study the prediction for leptogenesis within the framework of SM and MSSM. The condition of successful baryogenesis will allow us to determine the mass of the lightest right-handed neutrino in the new Littlest Seesaw model.

\subsection{Leptogenesis for the new Littlest Seesaw model in the SM}

In the SM, the final baryon asymmetry is given by [65]

$$
Y_{B}=\frac{12}{37} \sum_{\alpha} Y_{\Delta_{\alpha}}
$$

where the asymmetries $Y_{\Delta_{\alpha}}(\alpha=e, \mu, \tau)$ are defined as $Y_{\Delta_{\alpha}} \equiv Y_{B} / 3-Y_{L_{\alpha}}$ and they are conserved by the sphaleron processes [66]. $Y_{L_{\alpha}}$ refers to the lepton number densities of the flavour $\alpha$. Note that $Y_{B}, Y_{\Delta_{\alpha}}$ and $Y_{L_{\alpha}}$ is normalised to the entropy density. 
In the present work, we shall discuss the flavoured thermal leptogenesis scenario in $2 \mathrm{RHN}$ model with hierarchical Majorana masses $\left(M_{1} \ll M_{2}\right)$, where the two right-handed neutrino masses $M_{1}=x_{\text {atm }}\left\langle\xi_{\text {atm }}\right\rangle$ and $M_{2}=x_{\text {sol }}\left\langle\xi_{\text {sol }}\right\rangle$ are the masses of the right-handed neutrinos $N_{\text {atm }}$ and $N_{\text {sol }}$, respectively. The flavoured thermal leptogenesis has been studied in detail [65-67]. It was shown that the Boltzmann equations describing the asymmetries in flavour space are given by [68]

$$
\begin{array}{ll}
\frac{\mathrm{d} Y_{N_{\mathrm{atm}}}}{\mathrm{d} z} & =K\left(Y_{N_{\mathrm{atm}}}^{\mathrm{eq}}-Y_{N_{\mathrm{atm}}}\right) \frac{z f_{1}(z) K_{1}(z)}{K_{2}(z)}, \\
\frac{\mathrm{d} Y_{\Delta_{\alpha}}}{\mathrm{d} z} & =\varepsilon_{1 \alpha}^{\mathrm{SM}} K\left(Y_{N_{\mathrm{atm}}}^{\mathrm{eq}}-Y_{N_{\mathrm{atm}}}\right) \frac{z f_{1}(z) K_{1}(z)}{K_{2}(z)}+K_{\alpha} Y_{N_{\mathrm{atm}}}^{\mathrm{eq}} \frac{z f_{2}(z) K_{1}(z)}{K_{2}(z)} \frac{\sum_{\gamma} A_{\alpha \gamma}^{\mathrm{SM}} Y_{\Delta_{\gamma}}}{Y_{\ell}^{\mathrm{eq}}} .
\end{array}
$$

There is no sum over $\alpha$ in the last term of Eq. (24), $z=M_{1} / T$ with $T$ being the temperature, $K_{1}(z)$ and $K_{2}(z)$ are the modified Bessel functions of the second kind, and $Y_{N_{\mathrm{atm}}}$ denotes the density of the lightest right-handed neutrino $N_{\mathrm{atm}}{ }^{3} \cdot Y_{N_{\mathrm{atm}}}^{\mathrm{eq}}$ and $Y_{\ell}^{\mathrm{eq}}$ stand for the corresponding equilibrium number densities and they take the following form

$$
Y_{\ell}^{\mathrm{eq}} \simeq \frac{45}{\pi^{4} g_{*}^{\mathrm{SM}}}, \quad Y_{N_{\mathrm{atm}}}^{\mathrm{eq}} \simeq \frac{45 z^{2} K_{2}(z)}{2 \pi^{4} g_{*}^{\mathrm{SM}}},
$$

with $g_{*}^{\mathrm{SM}}=106.75$. In order to obtain phenomenologically viable baryon asymmetry, the lighter right-handed neutrino mass $M_{1}$ is assumed in the interval of $10^{9} \mathrm{GeV} \leq M_{1} \leq 10^{12} \mathrm{GeV}$. In this scenario, the $\tau$ Yukawa interaction is in equilibrium, the $e$ and $\mu$ flavours are indistinguishable, and the lepton number densities and $Y_{\Delta_{\alpha}}$ in the $e$ and $\mu$ flavour can be combined to $Y_{2} \equiv Y_{e+\mu}$ and $Y_{\Delta_{2}} \equiv Y_{\Delta_{e}+\Delta_{\mu}}$ [65-67]. In this temperature range, the matrix $A^{\mathrm{SM}}$ in the Boltzmann equation Eq. (24) is given by [66]

$$
A^{\mathrm{SM}}=\frac{1}{589}\left(\begin{array}{cc}
-417 & 120 \\
30 & -390
\end{array}\right)
$$

which arises from the washout term. The functions $f_{1}(z)$ and $f_{2}(z)$ in Eqs. (23) and (24) account for the presence of $\Delta L=1$ scatterings and scatterings in the washout term of the asymmetry respectively $[69,70]$. In the strong washout regime, $f_{1}(z)$ and $f_{2}(z)$ can be approximated as $[69,70]$

$$
f_{1}(z)=2 f_{2}(z)=\left[\frac{K_{s}}{z K}+\frac{z}{t} \ln \left(1+\frac{t}{z}\right)\right] \frac{K_{2}(z)}{K_{1}(z)}
$$

with

$$
t=\frac{K}{K_{s} \ln \left(M_{1} / M_{h}\right)}, \quad \frac{K_{s}}{K}=\frac{9}{8 \pi^{2}} .
$$

where $M_{h}=125 \mathrm{GeV}$ is the mass of the Higgs boson. The flavoured CP asymmetries in the decays of the lightest RHN $N_{\text {atm }}$ into Higgs and leptons of different flavours are of the form [70-73]

$$
\varepsilon_{1 \alpha}^{\mathrm{SM}}=\frac{1}{8 \pi\left(\lambda \lambda^{\dagger}\right)_{11}}\left\{\Im\left[\left(\lambda \lambda^{\dagger}\right)_{12} \lambda_{1 \alpha} \lambda_{2 \alpha}^{*}\right] g^{\mathrm{SM}}(y)+\frac{1}{y-1} \Im\left[\left(\lambda \lambda^{\dagger}\right)_{21} \lambda_{1 \alpha} \lambda_{2 \alpha}^{*}\right]\right\}
$$

where $y=M_{2}^{2} / M_{1}^{2}, \lambda$ is the neutrino Yukawa coupling matrix and it is a $2 \times 3$ matrix with the following form

$$
\lambda=\left(\begin{array}{ccc}
0 & -a & a \\
b e^{-\frac{i \pi}{4}} & \frac{5}{2} b e^{-\frac{i \pi}{4}} & -\frac{1}{2} b e^{-\frac{i \pi}{4}}
\end{array}\right),
$$

\footnotetext{
${ }^{3}$ We find that the observed excess of matter over antimatter can not be generated in the new Littlest Seesaw model if $N_{\text {sol }}$ is the lightest right-handed neutrino.
} 
where $a=\left|y_{\text {atm }} v_{\text {atm }}\right| / v, b=\left|y_{\text {sol }} v_{\text {sol }}\right| / v$ and $v=246 / \sqrt{2} \mathrm{GeV}$ is the VEV of the Higgs field. The loop function $g^{\mathrm{SM}}(y)$ in Eq. (29) can be written as

$$
g^{\mathrm{SM}}(y)=\sqrt{y}\left[\frac{1}{1-y}+1-(1+y) \ln \left(\frac{1+y}{y}\right)\right] \stackrel{y \gg 1}{\longrightarrow}-\frac{3}{2 \sqrt{y}} .
$$

Since hierarchical RHN masses $M_{1} \ll M_{2}(y \gg 1)$ are assumed, we can get the following approximation formula for the decay asymmetry

$$
\varepsilon_{1 \alpha}^{\mathrm{SM}}=-\frac{3}{16 \pi} \frac{\Im\left[\left(\lambda \lambda^{\dagger}\right)_{12} \lambda_{1 \alpha} \lambda_{2 \alpha}^{*}\right]}{\left(\lambda \lambda^{\dagger}\right)_{11}} \frac{M_{1}}{M_{2}} .
$$

For the breaking pattern discussed in section 2, the flavour dependent decay asymmetries are:

$$
\varepsilon_{1 e}^{\mathrm{SM}}=0, \quad \varepsilon_{1 \mu}^{\mathrm{SM}}=\frac{3}{16 \pi} \frac{M_{1}}{M_{2}}(x-1)(x-2) b^{2} \sin \eta, \quad \varepsilon_{1 \tau}^{\mathrm{SM}}=\frac{3}{16 \pi} \frac{M_{1}}{M_{2}} x(x-1) b^{2} \sin \eta .
$$

In the new Littlest Seesaw model with $x=-1 / 2, \eta=-\pi / 2, \varepsilon_{1 \alpha}^{\mathrm{SM}}(\alpha=e, \mu, \tau)$ read as

$$
\varepsilon_{1 e}^{\mathrm{SM}}=0, \quad \varepsilon_{1 \mu}^{\mathrm{SM}}=-\frac{45}{64 \pi} \frac{M_{1}}{M_{2}} b^{2}, \quad \varepsilon_{1 \tau}^{\mathrm{SM}}=-\frac{9}{64 \pi} \frac{M_{1}}{M_{2}} b^{2} .
$$

Note that $b^{2} / M_{2} \propto m_{s}$ which is defined in Eq. (1), once the value of $m_{s}$ is fixed through the masses squared differences $\Delta m_{21}^{2}$ and $\Delta m_{31}^{2}, \varepsilon_{1 \mu}^{\mathrm{SM}}$ and $\varepsilon_{1 \tau}^{\mathrm{SM}}$ only depend on the lightest right-handed neutrino mass $M_{1}$. In addition to the decay asymmetry, the washout parameter $K_{\alpha}$, which appears in the washout term of the Boltzmann equation, is given by

$$
K_{\alpha}=\frac{\widetilde{m}_{1 \alpha}}{m_{\mathrm{SM}}^{*}}, \quad K=\sum_{\alpha} K_{\alpha}
$$

where $m_{\mathrm{SM}}^{*} \simeq 1.08 \times 10^{-3} \mathrm{eV}$ and the washout mass $\widetilde{m}_{1 \alpha}$ parameterizes the decay rate of $N_{\text {atm }}$ into the leptons of flavour $\alpha$ with

$$
\widetilde{m}_{1 \alpha} \equiv \frac{\left|\lambda_{1 \alpha}\right|^{2} v^{2}}{M_{1}}
$$

From the Yukawa coupling matrix $\lambda$ given in Eq. (30), we find $K_{\alpha}$ is given by

$$
K_{e}=0, \quad K_{\mu}=K_{\tau}=\frac{m_{a}}{m_{\mathrm{SM}}^{*}} .
$$

where $m_{a}=a^{2} v^{2} / M_{1}$ is defined in Eq. (1). For the new Littlest Seesaw model, the best fitting value of $m_{a}$ is $m_{a}=22.366 \mathrm{meV}^{4}$ as shown in table 1 . Then we can obtain the washout parameters $K_{\alpha}$ as follows

$$
K_{e}+K_{\mu}=20.709 \gg 1, \quad K_{\tau}=20.709 \gg 1 .
$$

Hence all flavours are in the strong washout region. Numerically solving the Boltzmann equations in Eqs. $(23,24)$, we find that the observed baryon asymmetry $Y_{B}=8.7 \times 10^{-11}$ fix the lightest right-handed neutrino mass in the new Littlest Seesaw model:

$$
M_{1}=1.176 \times 10^{11} \mathrm{GeV} .
$$

We plot the baryon asymmetry $Y_{B}$ with respect to the Dirac CP phase $\delta_{C P}$ in figure 4 . The width of the line comes from varying $m_{a}$ and $r$ over their allowed ranges, where all three mixing angles and two neutrino mass squared differences are required to lie in the experimentally preferred $3 \sigma$ ranges [8].

\footnotetext{
${ }^{4}$ The parameter $m_{a}$ should be in the range $21.707 \mathrm{meV} \leq m_{a} \leq 23.019 \mathrm{meV}$ in order to be compatible with present neutrino oscillation data.
} 


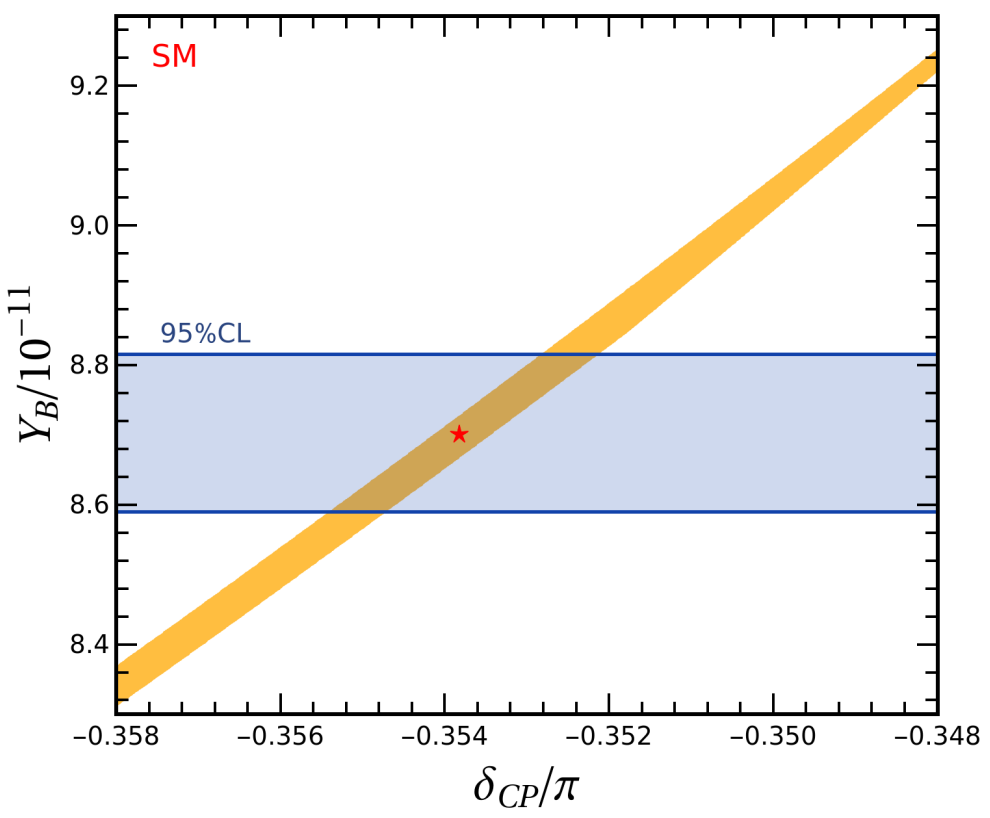

Figure 4: The correlation between $Y_{B}$ and $\delta_{C P}$ for the new Littlest Seesaw model in SM where $M_{1}=1.176 \times 10^{11} \mathrm{GeV}$. The Planck result for the baryon asymmetry $Y_{B}$ at $95 \%$ CL is represented by the horizontal band [63]. The red star denotes the best fitting point at which the $\chi^{2}$ function reaches a global minimum.

\subsection{Leptogenesis for the new Littlest Seesaw model in the MSSM}

In the MSSM, the final baryon asymmetry can be computed from the following formula [74]

$$
Y_{B}=\frac{10}{31} \sum_{\alpha} \hat{Y}_{\Delta_{\alpha}}
$$

In the MSSM, the contributions of $\widetilde{N}_{1}$ and $\widetilde{L}_{\alpha}$ should be considered, which are the supersymmetric partners of the lightest right-handed neutrino $N_{1}$ and the lepton doublet $L_{\alpha}$ respectively. In other words, the densities $Y_{\widetilde{N}_{1}}$ and $Y_{\widetilde{\alpha}}$ should be included in the Boltzmann equations. Then the Boltzmann equations in MSSM are given by [68]

$$
\begin{aligned}
\frac{\mathrm{d} Y_{N_{\mathrm{atm}}}}{\mathrm{d} z}= & 2 K\left(Y_{N_{\mathrm{atm}}}^{\mathrm{eq}}-Y_{N_{\mathrm{atm}}}\right) \frac{z f_{1}(z) K_{1}(z)}{K_{2}(z)} \\
\frac{\mathrm{d} Y_{\widetilde{N}_{\mathrm{atm}}}}{\mathrm{d} z}= & 2 K\left(Y_{\widetilde{N}_{\mathrm{atm}}^{\mathrm{eq}}}^{\mathrm{d}}-Y_{\widetilde{N}_{\mathrm{atm}}}\right) \frac{z f_{1}(z) K_{1}(z)}{K_{2}(z)} \\
\frac{\mathrm{d} \hat{Y}_{\Delta_{\alpha}}}{\mathrm{d} z}= & K\left(\varepsilon_{1 \alpha}^{\mathrm{MSSM}}+\varepsilon_{1 \widetilde{\alpha}}^{\mathrm{MSSM}}\right)\left(Y_{N_{\mathrm{atm}}}^{\mathrm{eq}}-Y_{N_{\mathrm{atm}}}\right) \frac{z f_{1}(z) K_{1}(z)}{K_{2}(z)} \\
& +K\left(\varepsilon_{\widetilde{1} \alpha}^{\mathrm{MSSM}}+\varepsilon_{\widetilde{1} \widetilde{\alpha}}^{\mathrm{MSSM}}\right)\left(Y_{\widetilde{N}_{\mathrm{atm}}^{\mathrm{eq}}}^{\mathrm{eq}}-Y_{\widetilde{N}_{\mathrm{atm}}}\right) \frac{z f_{1}(z) K_{1}(z)}{K_{2}(z)} \\
& +K_{\alpha}\left(Y_{N_{\mathrm{atm}}}^{\mathrm{eq}}+Y_{\widetilde{N}_{\mathrm{atm}}}^{\mathrm{eq}}\right) \frac{z f_{2}(z) K_{1}(z)}{K_{2}(z)} \frac{\sum_{\gamma} A_{\alpha \gamma}^{\mathrm{MSSM}} \hat{Y}_{\Delta_{\gamma}}}{\hat{Y}_{\ell}^{\mathrm{eq}}},
\end{aligned}
$$

where the total (particle and sparticle) $B / 3-L_{\alpha}$ asymmetries denoted as $\hat{Y}_{\Delta_{\alpha}}$ and

$$
\hat{Y}_{\ell}^{\mathrm{eq}}=Y_{\tilde{\ell}}^{\mathrm{eq}}+Y_{\ell}^{\mathrm{eq}}, \quad Y_{\tilde{\ell}}^{\mathrm{eq}} \simeq Y_{\ell}^{\mathrm{eq}} \simeq \frac{45}{\pi^{4} g_{*}^{\mathrm{MSSM}}}, \quad Y_{N_{\mathrm{atm}}}^{\mathrm{eq}}=Y_{\widetilde{N}_{\mathrm{atm}}}^{\mathrm{eq}} \simeq \frac{45 z^{2} K_{2}(z)}{2 \pi^{4} g_{*}^{\mathrm{MSSM}}},
$$


with $g_{*}^{\mathrm{MSSM}}=228.75$. The matrix $A^{\mathrm{MSSM}}$ in Eq. (41) depends on which MSSM interactions are in thermal equilibrium at the temperatures where leptogenesis takes place. Here we shall consider the case that the lightest right-handed neutrino mass $M_{1}$ is between $\left(1+\tan ^{2} \beta\right) \times 10^{9} \mathrm{GeV}$ and $\left(1+\tan ^{2} \beta\right) \times 10^{12} \mathrm{GeV}$, where only the $\tau$ Yukawa couplings are in thermal equilibrium. Then the relevant flavour-dependent asymmetries are $\hat{Y}_{\Delta_{2}} \equiv \hat{Y}_{\Delta_{e}+\Delta_{\mu}}$ and $\hat{Y}_{\Delta_{\tau}}$, and $A^{\mathrm{MSSM}}$ is given by

$$
A^{\mathrm{MSSM}}=\frac{1}{761}\left(\begin{array}{rr}
-541 & 152 \\
46 & -494
\end{array}\right) \text {. }
$$

In the MSSM, the decay asymmetries are all equal $\left(\varepsilon_{1 \alpha}^{\mathrm{MSSM}}=\varepsilon_{1 \widetilde{\alpha}}^{\mathrm{MSSM}}=\varepsilon_{\widetilde{1} \alpha}^{\mathrm{MSSM}}=\varepsilon_{\widetilde{1} \widetilde{\alpha}}^{\mathrm{MSS}}\right)[71]$. As a consequence, we will only show the results of $\varepsilon_{1 \alpha}^{\mathrm{MSSM}}$ in the following. Under the assumption of $M_{1} \ll M_{2}$, the CP asymmetry $\varepsilon_{1 \alpha}^{\mathrm{MSSM}}(\alpha=e, \mu, \tau)$ in the MSSM is given by

$$
\varepsilon_{1 \alpha}^{\mathrm{MSSM}}=\frac{1}{8 \pi\left(\lambda \lambda^{\dagger}\right)_{11}} \Im\left[\left(\lambda \lambda^{\dagger}\right)_{12} \lambda_{1 \alpha} \lambda_{2 \alpha}^{*}\right] g^{\mathrm{MSSM}}\left(\frac{M_{2}^{2}}{M_{1}^{2}}\right)
$$

where the function $g^{\operatorname{MSSM}}(y)$ is of the following form

$$
g^{\operatorname{MSSM}}(y)=\sqrt{y}\left[\frac{2}{1-y}-\ln \left(\frac{1+y}{y}\right)\right] \stackrel{y \gg 1}{\longrightarrow}-\frac{3}{\sqrt{y}} .
$$

Inserting the expression of function $g^{\mathrm{MSSM}}\left(M_{2}^{2} / M_{1}^{2}\right)$ into $\varepsilon_{1 \alpha}^{\mathrm{MSSM}}$ in Eq. (44) we find

$$
\varepsilon_{1 e}^{\mathrm{MSSM}}=0, \quad \varepsilon_{1 \mu}^{\mathrm{MSSM}}=\frac{3}{8 \pi} \frac{M_{1}}{M_{2}}(x-1)(x-2) b^{2} \sin \eta, \quad \varepsilon_{1 \tau}^{\mathrm{MSSM}}=\frac{3}{8 \pi} \frac{M_{1}}{M_{2}} x(x-1) b^{2} \sin \eta,
$$

for the most general case. In the new Littlest Seesaw model with $x=-1 / 2, \eta=-\pi / 2$, the flavour dependent decay asymmetries are determined to be,

$$
\varepsilon_{1 e}^{\mathrm{MSSM}}=0, \quad \varepsilon_{1 \mu}^{\mathrm{MSSM}}=-\frac{45}{32 \pi} \frac{M_{1}}{M_{2}} b^{2}, \quad \varepsilon_{1 \tau}^{\mathrm{MSSM}}=-\frac{9}{32 \pi} \frac{M_{1}}{M_{2}} b^{2} .
$$

The washout parameters $K_{\alpha}$ and $K$ in Eq. (41) are defined as

$$
K_{\alpha}=\frac{\tilde{m}_{1 \alpha}}{m_{\mathrm{MSSM}}^{*}}, \quad \tilde{m}_{1 \alpha} \equiv \frac{\left|\lambda_{1 \alpha}\right|^{2} v_{u}^{2}}{M_{1}}, \quad K=\sum_{\alpha} K_{\alpha},
$$

with

$$
v_{u}=v \sin \beta, \quad m_{\mathrm{MSSM}}^{*} \simeq \sin ^{2} \beta \times 1.58 \times 10^{-3} \mathrm{eV} .
$$

The expressions of the washout parameters for the new Littlest Seesaw model are

$$
K_{e}=0, \quad K_{\mu}=K_{\tau}=\frac{m_{a}}{m_{\mathrm{MSSM}}^{*}},
$$

with $m_{a}=a^{2} v_{u}^{2} / M_{1}$. At the best fitting of our model, the values of the washout parameters are

$$
K_{e}+K_{\mu}=14.722 \gg 1, \quad K_{\tau}=14.722 \gg 1
$$

which implies all flavours are in the strong washout region. For illustration, we take $\tan \beta=5$ and we find the experimentally observed values of the baryon asymmetry can be obtained if the lightest right-handed neutrino mass in the new Littlest Seesaw model is

$$
M_{1}=3.992 \times 10^{10} \mathrm{GeV} .
$$

The correlation between $Y_{B}$ and $\delta_{C P}$ in the new Littlest Seesaw model is displayed in figure 5 . 


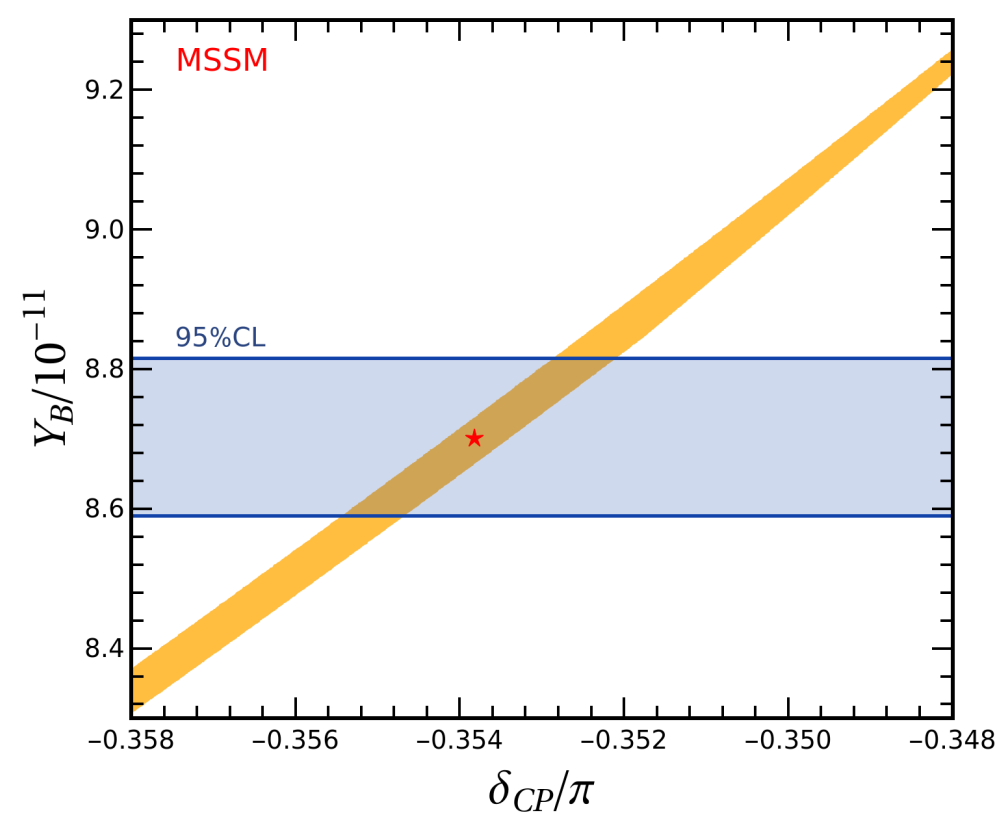

Figure 5: The correlation between $Y_{B}$ and $\delta_{C P}$ for the new Littlest Seesaw model in the MSSM where we take $M_{1}=3.992 \times 10^{10} \mathrm{GeV}$. The Planck result for the baryon asymmetry $Y_{B}$ at $95 \%$ CL is represented by the horizontal band [63]. The red star denotes the best fitting point at which the $\chi^{2}$ function reaches a global minimum.

\section{Explicit model for the new Littlest Seesaw}

As we have shown in previous sections, the new Littlest Seesaw model can describe the experiment data of lepton mixing angles, neutrino masses and matter-antimatter asymmetry of the Universe very well. In this section, we shall construct an explicit model based on the model independent analysis of section 2 . The vacuum alignments $\left\langle\phi_{\text {atm }}\right\rangle \propto(0,1,-1),\left\langle\phi_{\text {sol }}\right\rangle \propto(1,-1 / 2,5 / 2)$ and the phase parameter $\eta=-\pi / 2$ will be naturally realized in our model. We impose the $S_{4}$ flavour symmetry as well as CP symmetry. The standard supersymmetric driving field mechanism [75] which we adopt in our model requires a $U(1)_{R}$ symmetry related to the usual $R$-parity. Furthermore, we also introduce the shaping symmetry $Z_{5} \times Z_{8}$ which allows us to forbid unwanted terms and achieve the desired vacuum alignment. The auxiliary symmetry $Z_{8}$ is helpful to generate the phase $\eta=-\pi / 2$. The shaping symmetry $Z_{5}$ requires the electron, muon and tauon mass terms couple with different powers of flavon fields. Hence $Z_{5}$ helps to reproduce the observed charged lepton mass hierarchies. Here we choose the right-handed charged leptons as $S_{4}$ singlets, where $e^{c}$ and $\tau^{c}$ transform as $\mathbf{1}$ while $\mu^{c}$ transforms as $\mathbf{1}^{\prime}$. The three generations of left-handed lepton doublets $L$ are unified to an $S_{4}$ triplet 3 . The two right-handed neutrinos $\nu_{\text {atm }}^{c}$ and $\nu_{\text {sol }}^{c}$ are assigned to 1 and $\mathbf{1}^{\prime}$ of $S_{4}$, respectively. The field content and their classification under the flavour symmetry $S_{4} \times Z_{5} \times Z_{8}$ are listed in table 2 . The driving fields are indicated with the superscript " 0 " and they carry two units of $R$ charge, both flavon fields and Higgs are uncharged under $U(1)_{R}$, and the $R$-charge of the matter fields is equal to one. Since both flavon fields and driving fields are Standard Model singlets, our model is anomaly free under the Standard Model gauge transformation. As regards possible discrete anomalies, in principle they may be cancelled by adding extra states under the discrete group, but this is beyond the scope of this paper. In the following, we first discuss the vacuum alignment of the model, then specify the structure of the model. 


\begin{tabular}{|c|c|c|c|c|c|c|c||c|c||c|c|c|c||c|c|c||c|c|c||c|c|c|c|c|c|c||c|}
\hline \hline & $L$ & $e^{c}$ & $\mu^{c}$ & $\tau^{c}$ & $\nu_{\text {atm }}^{c}$ & $\nu_{\text {sol }}^{c}$ & $H_{u, d}$ & $\chi_{l}$ & $\phi_{l}$ & $\xi_{a}$ & $\zeta_{a}$ & $\eta_{a}$ & $\phi_{a}$ & $\xi_{s}$ & $\varphi_{s}$ & $\phi_{s}$ & $\eta_{l}^{0}$ & $\xi_{l}^{0}$ & $\zeta_{l}^{0}$ & $\xi_{a}^{0}$ & $\eta_{a}^{0}$ & $\phi_{a}^{0}$ & $\xi_{s}^{0}$ & $\varphi_{s}^{0}$ & $\zeta_{s}^{0}$ & $\phi_{s}^{0}$ & $\sigma_{1,2}^{0}$ \\
\hline$S_{4}$ & $\mathbf{3}$ & $\mathbf{1}$ & $\mathbf{1}^{\prime}$ & $\mathbf{1}$ & $\mathbf{1}$ & $\mathbf{1}^{\prime}$ & $\mathbf{1}$ & $\mathbf{3}$ & $\mathbf{3}$ & $\mathbf{1}$ & $\mathbf{1}^{\prime}$ & $\mathbf{2}$ & $\mathbf{3}$ & $\mathbf{1}$ & $\mathbf{3}$ & $\mathbf{3}^{\prime}$ & $\mathbf{2}$ & $\mathbf{1}$ & $\mathbf{1}$ & $\mathbf{1}$ & $\mathbf{2}$ & $\mathbf{3}^{\prime}$ & $\mathbf{1}$ & $\mathbf{3}^{\prime}$ & $\mathbf{1}^{\prime}$ & $\mathbf{3}^{\prime}$ & $\mathbf{1}$ \\
$Z_{5}$ & 1 & $\omega_{5}^{2}$ & $\omega_{5}^{3}$ & $\omega_{5}^{4}$ & 1 & 1 & 1 & $\omega_{5}^{4}$ & $\omega_{5}$ & 1 & $\omega_{5}^{4}$ & 1 & 1 & 1 & $\omega_{5}$ & 1 & $\omega_{5}^{2}$ & $\omega_{5}^{3}$ & 1 & $\omega_{5}$ & 1 & $\omega_{5}$ & 1 & $\omega_{5}^{4}$ & $\omega_{5}^{4}$ & $\omega_{5}^{4}$ & 1 \\
$Z_{8}$ & 1 & $\omega_{8}^{3}$ & $i$ & $\omega_{8}$ & $-i$ & $\omega_{8}^{5}$ & 1 & $i$ & $\omega_{8}^{7}$ & -1 & -1 & -1 & $i$ & $-i$ & $-i$ & $\omega_{8}^{3}$ & -1 & $i$ & $\omega_{8}^{7}$ & -1 & -1 & $i$ & $i$ & 1 & $\omega_{8}^{7}$ & $\omega_{8}^{7}$ & 1 \\
\hline \hline
\end{tabular}

Table 2: The matter field, flavon fields, driving fields and their transformation properties under the flavour symmetry $S_{4} \times Z_{5} \times Z_{8}$ in model, where $\omega_{5}=e^{2 \pi i / 5}$ and $\omega_{8}=e^{\pi i / 4}$.

\subsection{Vacuum alignment}

We employ the now-standard $F$-term alignment mechanism to arrange the vacuum [75] in our model. It requires that all terms in the superpotential must carry two units of $R$ charge. Therefore each term in the superpotential contains either two matter superfields or only one driving field. The minimum of the scalar potential is determined by vanishing $F$-terms of the driving fields. The leading order (LO) driving superpotential $w_{d}$ in which each term contains one driving field invariant under $S_{4} \times Z_{5} \times Z_{8}$ can be written as

$$
\begin{aligned}
w_{d}= & f_{1}\left(\eta_{l}^{0}\left(\chi_{l} \chi_{l}\right)_{\mathbf{2}}\right)_{\mathbf{1}}+f_{2} \xi_{l}^{0}\left(\phi_{l} \phi_{l}\right)_{\mathbf{1}}+f_{3} \zeta_{l}^{0}\left(\phi_{l} \chi_{l}\right)_{\mathbf{1}}+f_{4} \xi_{a}^{0}\left(\chi_{l} \phi_{a}\right)_{\mathbf{1}}+M_{\eta}\left(\eta_{a}^{0} \eta_{a}\right)_{\mathbf{1}} \\
& +f_{5}\left(\eta_{a}^{0}\left(\phi_{a} \phi_{a}\right)_{\mathbf{2}}\right)_{\mathbf{1}}+f_{6} \zeta_{a}\left(\phi_{a}^{0} \phi_{a}\right)_{\mathbf{1}^{\prime}}+f_{7}\left(\phi_{a}^{0}\left(\eta_{a} \chi_{l}\right)_{\mathbf{3}^{\prime}}\right)_{\mathbf{1}}+M_{\sigma_{1}}^{2} \sigma_{1}^{0}+f_{8} \sigma_{1}^{0} \xi_{a}^{2} \\
& +M_{\sigma_{2}}^{2} \sigma_{2}^{0}+f_{9} \sigma_{2}^{0}\left(\eta_{a} \eta_{a}\right)_{\mathbf{1}}+f_{10}\left(\varphi_{s}^{0}\left(\phi_{a} \varphi_{s}\right)_{\mathbf{3}^{\prime}}\right)_{\mathbf{1}}+f_{11} \zeta_{s}^{0}\left(\varphi_{s} \phi_{s}\right)_{\mathbf{1}^{\prime}}+f_{12}\left(\phi_{s}^{0}\left(\phi_{l} \phi_{a}\right)_{\mathbf{3}^{\prime}}\right)_{\mathbf{1}} \\
& +f_{13}\left(\phi_{s}^{0}\left(\varphi_{s} \phi_{s}\right)_{\mathbf{3}^{\prime}}\right)_{\mathbf{1}}+M_{\xi} \xi_{s}^{0} \xi_{s}+f_{14} \xi_{s}^{0}\left(\phi_{s} \phi_{s}\right)_{\mathbf{1}},
\end{aligned}
$$

where $(\ldots)_{\mathbf{r}}$ stands for a contraction into the $S_{4}$ irreducible representation $\mathbf{r}$. Because we impose $\mathrm{CP}$ as symmetry on the model, all the couplings $f_{i}(i=1, \cdots, 14)$ and mass parameters $M_{\eta}, M_{\xi}$, $M_{\sigma_{1}}, M_{\sigma_{2}}$ are constrained to be real. The VEVs of the flavon $\chi_{l}$ can be obtained from the vanishing of the derivatives of $w_{d}$ with respect to each component of the driving fields $\eta_{l}^{0}$, i.e.

$$
\begin{aligned}
& \frac{\partial w_{d}}{\partial \eta_{l_{1}}^{0}}=f_{1}\left(2 \chi_{l_{1}} \chi_{l_{2}}+\chi_{l_{3}}^{2}\right)=0 \\
& \frac{\partial w_{d}}{\partial \eta_{l_{2}}^{0}}=f_{1}\left(2 \chi_{l_{1}} \chi_{l_{3}}+\chi_{l_{2}}^{2}\right)=0
\end{aligned}
$$

One solution to these equations is

$$
\left\langle\chi_{l}\right\rangle=v_{\chi_{l}}(1,0,0)^{T},
$$

where $v_{\chi_{l}}$ is undetermined. In the charged lepton sector, the $F$-term conditions of the driving fields $\xi_{l}^{0}$ and $\zeta_{l}^{0}$ give the vacuum alignment of $\phi_{l}$,

$$
\begin{aligned}
& \frac{\partial w_{d}}{\partial \xi_{l}^{0}}=f_{2}\left(\phi_{l_{1}}^{2}+2 \phi_{l_{2}} \phi_{l_{3}}\right)=0, \\
& \frac{\partial w_{d}}{\partial \zeta_{l}^{0}}=f_{3}\left(\chi_{l_{1}} \phi_{l_{1}}+\chi_{l_{2}} \phi_{l_{3}}+\chi_{l_{3}} \phi_{l_{2}}\right)=0 .
\end{aligned}
$$

Given the vacuum of $\chi_{l}$ in Eq. (55), we find the alignment of $\phi_{l}$ is

$$
\left\langle\phi_{l}\right\rangle=v_{\phi_{l}}(0,1,0)^{T}
$$


with $v_{\phi_{l}}$ undetermined. In the atmospheric neutrino sector, the $F$-term conditions associated with the driving fields $\xi_{a}^{0}, \eta_{a}^{0}$ and $\phi_{a}^{0}$ read

$$
\begin{aligned}
\frac{\partial w_{d}}{\partial \xi_{a}^{0}} & =f_{4}\left(\chi_{l_{1}} \phi_{a_{1}}+\chi_{l_{2}} \phi_{a_{3}}+\chi_{l_{3}} \phi_{a_{2}}\right)=0, \\
\frac{\partial w_{d}}{\partial \eta_{a_{1}}^{0}} & =M_{\eta} \eta_{a_{2}}+f_{5}\left(2 \phi_{a_{1}} \phi_{a_{2}}+\phi_{a_{3}}^{2}\right)=0, \\
\frac{\partial w_{d}}{\partial \eta_{a_{2}}^{0}} & =M_{\eta} \eta_{a_{1}}+f_{5}\left(2 \phi_{a_{1}} \phi_{a_{3}}+\phi_{a_{2}}^{2}\right)=0, \\
\frac{\partial w_{d}}{\partial \phi_{a_{1}}^{0}} & =f_{6} \zeta_{a} \phi_{a_{1}}+f_{7}\left(\eta_{a_{1}} \chi_{l_{2}}-\eta_{a_{2}} \chi_{l_{3}}\right)=0, \\
\frac{\partial w_{d}}{\partial \phi_{a_{2}}^{0}} & =f_{6} \zeta_{a} \phi_{a_{3}}+f_{7}\left(\eta_{a_{1}} \chi_{l_{1}}-\eta_{a_{2}} \chi_{l_{2}}\right)=0, \\
\frac{\partial w_{d}}{\partial \phi_{a_{3}}^{0}} & =f_{6} \zeta_{a} \phi_{a_{2}}+f_{7}\left(\eta_{a_{1}} \chi_{l_{3}}-\eta_{a_{2}} \chi_{l_{1}}\right)=0 .
\end{aligned}
$$

A straightforward calculation shows that the vacuum expectation values of $\xi_{a}, \eta_{a}$ and $\phi_{a}$ are

$$
\left\langle\zeta_{a}\right\rangle=v_{\zeta_{a}}, \quad\left\langle\eta_{a}\right\rangle=v_{\eta_{a}}(1,1)^{T}, \quad\left\langle\phi_{a}\right\rangle=v_{\phi_{a}}(0,1,-1)^{T},
$$

with

$$
v_{\phi_{a}}^{2}=-\frac{M_{\eta}}{f_{5}} v_{\eta_{a}}, \quad v_{\zeta_{a}}=-\frac{f_{5} f_{7} v_{\phi_{a}} v_{\chi_{l}}}{f_{6} M_{\eta}}
$$

It is easy to check that the vacuum alignments of flavons $\eta_{a}$ and $\phi_{a}$ preserve the subgroup $Z_{2}^{U}$. Now we consider the phases of $v_{\phi_{a}}$ and $v_{\xi_{a}}$ which is the VEV of $\xi_{a}$. They are related by the $F$-flatness of $\sigma_{1,2}^{0}$ :

$$
\begin{aligned}
& \frac{\partial w_{d}}{\partial \sigma_{1}^{0}}=M_{\sigma_{1}}^{2}+f_{8} \xi_{a}^{2}=0, \\
& \frac{\partial w_{d}}{\partial \sigma_{2}^{0}}=M_{\sigma_{2}}^{2}+2 f_{9} \eta_{a_{1}} \eta_{a_{2}}=0 .
\end{aligned}
$$

From Eqs. (60) and (61), we find

$$
\frac{v_{\xi_{a}}}{v_{\phi_{a}}^{2}}=\frac{f_{5} M_{\sigma_{1}}}{M_{\sigma_{2}} M_{\eta}}\left(\frac{2 f_{9}}{f_{8}}\right)^{1 / 2} .
$$

As all parameters in the right-hand side of above equation are real, consequently the phase of $\frac{v_{\xi_{a}}}{v_{\phi_{a}}^{2}}$ is $0, \pi$ or $\pm \pi / 2$ for the product $f_{8} f_{9}>0$ or $f_{8} f_{9}<0$, respectively. The auxiliary symmetry $Z_{8}$ has played a critical role in generating the discrete possible values $0, \pi, \pm \pi / 2$ for the phase of $v_{\xi_{a}} / v_{\phi_{a}}^{2}$. Subsequently we turn to discuss the vacuum alignment of the solar neutrino sector. The $F$-flatness condition of the driving field $\varphi_{s}^{0}$ gives

$$
\begin{aligned}
\frac{\partial w_{d}}{\partial \varphi_{s_{1}}^{0}} & =f_{10}\left(2 \varphi_{s_{1}} \phi_{a_{1}}-\varphi_{s_{2}} \phi_{a_{3}}-\varphi_{s_{3}} \phi_{a_{2}}\right)=0, \\
\frac{\partial w_{d}}{\partial \varphi_{s_{2}}^{0}} & =f_{10}\left(2 \varphi_{s_{2}} \phi_{a_{2}}-\varphi_{s_{1}} \phi_{a_{3}}-\varphi_{s_{3}} \phi_{a_{1}}\right)=0, \\
\frac{\partial w_{d}}{\partial \varphi_{s_{3}}^{0}} & =f_{10}\left(2 \varphi_{s_{3}} \phi_{a_{3}}-\varphi_{s_{1}} \phi_{a_{2}}-\varphi_{s_{2}} \phi_{a_{1}}\right)=0,
\end{aligned}
$$


which lead to the vacuum

$$
\left\langle\varphi_{s}\right\rangle=v_{\varphi_{s}}(2,-1,-1)^{T} .
$$

The equations giving the vacuum structure for the flavon field $\phi_{s}$ are:

$$
\begin{aligned}
& \frac{\partial w_{d}}{\partial \zeta_{s}^{0}}=f_{11}\left(\varphi_{s_{1}} \phi_{s_{1}}+\varphi_{s_{2}} \phi_{s_{3}}+\varphi_{s_{3}} \phi_{s_{2}}\right)=0, \\
& \frac{\partial w_{d}}{\partial \phi_{s_{1}}^{0}}=f_{12}\left(2 \phi_{a_{1}} \phi_{l_{1}}-\phi_{a_{2}} \phi_{l_{3}}-\phi_{a_{3}} \phi_{l_{2}}\right)+f_{13}\left(\varphi_{s_{2}} \phi_{s_{3}}-\varphi_{s_{3}} \phi_{s_{2}}\right)=0, \\
& \frac{\partial w_{d}}{\partial \phi_{s_{2}}^{0}}=f_{12}\left(2 \phi_{a_{2}} \phi_{l_{2}}-\phi_{a_{1}} \phi_{l_{3}}-\phi_{a_{3}} \phi_{l_{1}}\right)+f_{13}\left(\varphi_{s_{3}} \phi_{s_{1}}-\varphi_{s_{1}} \phi_{s_{3}}\right)=0, \\
& \frac{\partial w_{d}}{\partial \phi_{s_{3}}^{0}}=f_{12}\left(2 \phi_{a_{3}} \phi_{l_{3}}-\phi_{a_{1}} \phi_{l_{2}}-\phi_{a_{2}} \phi_{l_{1}}\right)+f_{13}\left(\varphi_{s_{1}} \phi_{s_{2}}-\varphi_{s_{2}} \phi_{s_{1}}\right)=0,
\end{aligned}
$$

which uniquely determine the solar alignment,

$$
\left\langle\phi_{s}\right\rangle=v_{\phi_{s}}(1,-1 / 2,5 / 2)^{T}, \quad \text { with } \quad v_{\phi_{s}}=\frac{f_{12} v_{\phi_{l}} v_{\phi_{a}}}{3 f_{13} v_{\varphi_{s}}} .
$$

We find the vacuum configurations of $\varphi_{s}$ and $\phi_{s}$ are invariant under the action of the subgroup $Z_{2}^{S U}$. Finally the $F$-term condition of $\xi_{s}^{0}$ is

$$
\frac{\partial w_{d}}{\partial \xi_{s}^{0}}=M_{\xi} \xi_{s}+f_{14}\left(\phi_{s_{1}}^{2}+2 \phi_{s_{2}} \phi_{s_{3}}\right)=0
$$

which leads to the following relations

$$
\frac{v_{\phi_{s}}^{2}}{v_{\xi_{s}}}=\frac{2 M_{\xi}}{3 f_{14}}
$$

The phase parameter $\eta$ is exactly the phase of the ratio $\frac{v_{\phi_{s}}^{2} v_{\xi_{a}}}{v_{\phi_{a}}^{2} v_{\xi_{s}}}$ in our model. Form Eqs. (60) and (68), it is easy to obtain

$$
\frac{v_{\phi_{s}}^{2} v_{\xi_{a}}}{v_{\phi_{a}}^{2} v_{\xi_{s}}}=\frac{2 f_{5} M_{\sigma_{1}} M_{\xi}}{3 f_{14} M_{\sigma_{2}} M_{\eta}}\left(\frac{2 f_{9}}{f_{8}}\right)^{1 / 2} .
$$

All couplings and mass parameters in above equation are real due to $\mathrm{CP}$ symmetry, then we see the phase of the ratio $\frac{v_{\phi_{s}}^{2} v_{\xi_{a}}}{v_{\phi_{a}}^{2} v_{\xi_{s}}}$ is $e^{\frac{i k \pi}{2}}(i=0,1, \ldots, 3)$. In the present work we shall take the following solution

$$
\arg \left(\frac{v_{\phi_{s}}^{2} v_{\xi_{a}}}{v_{\phi_{a}}^{2} v_{\xi_{s}}}\right)=-\frac{\pi}{2},
$$

which would happen for $f_{8} f_{9}<0$. Thus the desired vacuum alignment $\left\langle\phi_{a}\right\rangle \propto(0,1,-1)^{T},\left\langle\phi_{s}\right\rangle \propto$ $(1,-1 / 2,5 / 2)^{T}$ and the phase $\eta=-\pi / 2$ have been dynamically realized. In the following section we will find that the observed hierarchy among the charged lepton masses can be produced for

$$
\frac{v_{\phi_{l}}}{\Lambda} \sim \lambda_{C}^{2},
$$

where $\Lambda$ is the cut-off scale of the theory and $\lambda_{C}$ is the Cabibbo angle with $\lambda_{C} \simeq 0.23$. As usual, we expect that all the VEVs of flavons are of the same order of magnitude, i.e.

$$
\frac{v_{\xi_{a}}}{\Lambda} \sim \frac{v_{\phi_{a}}}{\Lambda} \sim \frac{v_{\xi_{s}}}{\Lambda} \sim \frac{v_{\phi_{s}}}{\Lambda} \sim \frac{v_{\eta_{a}}}{\Lambda} \sim \frac{v_{\zeta_{a}}}{\Lambda} \sim \frac{v_{\varphi_{s}}}{\Lambda} \sim \frac{v_{\chi_{l}}}{\Lambda} \sim \lambda_{C}^{2}
$$

Successful leptogenesis fixes the atmospheric neutrino mass to be $3.992 \times 10^{10} \mathrm{GeV}$ (see Eq. (52)) which is of the same order as the flavon VEVs. Thus the cut-off scale $\Lambda$ is expected to be of order 
$10^{12} \mathrm{GeV}$. The next-to-leading-order (NLO) corrections to the flavon superpotential $w_{d}$ involve three flavon fields. When the NLO corrections are included, the original symmetry $S_{4} \rtimes H_{C P}$ is broken completely in the charged lepton, atmospheric neutrino and solar neutrino sectors. The NLO corrections to VEVs of all flavons are found to be suppressed by $\Phi / \Lambda \sim \lambda_{C}^{2}$ with respect to the LO contributions and therefore can be negligible, where $\Phi$ denotes any flavour fields.

\subsection{The structure of the model}

The most relevant operators for charged lepton masses are given by

$$
\begin{aligned}
w_{l} & =\frac{y_{\tau}}{\Lambda}\left(L \phi_{l}\right)_{\mathbf{1}} \tau^{c} H_{d}+\frac{y_{\mu}}{\Lambda^{2}}\left(L\left(\phi_{l} \phi_{l}\right)_{\mathbf{3}^{\prime}}\right)_{\mathbf{1}^{\prime}} \mu^{c} H_{d}+\frac{y_{e_{1}}}{\Lambda^{3}}\left(L \phi_{l}\right)_{\mathbf{1}}\left(\phi_{l} \phi_{l}\right)_{\mathbf{1}} e^{c} H_{d} \\
& +\frac{y_{e_{2}}}{\Lambda^{3}}\left(\left(L \phi_{l}\right)_{\mathbf{2}}\left(\phi_{l} \phi_{l}\right)_{\mathbf{2}}\right)_{\mathbf{1}} e^{c} H_{d}+\frac{y_{e_{3}}}{\Lambda^{3}}\left(\left(L \phi_{l}\right)_{\mathbf{3}}\left(\phi_{l} \phi_{l}\right)_{\mathbf{3}}\right)_{\mathbf{1}} e^{c} H_{d}+\frac{y_{e 4}}{\Lambda^{3}}\left(\left(L \phi_{l}\right)_{\mathbf{3}^{\prime}}\left(\phi_{l} \phi_{l}\right)_{\mathbf{3}^{\prime}}\right)_{\mathbf{1}} e^{c} H_{d},
\end{aligned}
$$

where all the couplings are real because of the CP symmetry. After the electroweak and $S_{4}$ flavour symmetry breaking by the VEV shown in Eq. (57), one can obtain that the charged lepton mass matrix is diagonal with the masses

$$
m_{e}=\left|\left(y_{e_{2}}-2 y_{e_{4}}\right) \frac{v_{\phi_{l}}^{3}}{\Lambda^{3}}\right| v_{d}, \quad m_{\mu}=\left|2 y_{\mu_{1}} \frac{v_{\phi_{l}}^{2}}{\Lambda^{2}}\right| v_{d}, \quad m_{\tau}=\left|y_{\tau} \frac{v_{\phi_{l}}}{\Lambda}\right| v_{d},
$$

where $v_{d}=\left\langle H_{d}\right\rangle$ is the VEV of the electroweak Higgs field $H_{d}$. Since the charged lepton mass matrix is diagonal, the hermitian combination $m_{l}^{\dagger} m_{l}$ is invariant under the action of the subgroup $Z_{3}^{T}$, i.e. $\rho_{\mathbf{3}}^{\dagger}(T) m_{l}^{\dagger} m_{l} \rho_{\mathbf{3}}(T)=m_{l}^{\dagger} m_{l}$. With the assignment in table 2 , the tau, muon and electron masses arise at the one-flavon, two-flavons and three-flavons level respectively in our model. Consequently the charged lepton mass hierarchies are naturally reproduced

$$
m_{e}: m_{\mu}: m_{\tau} \simeq \lambda_{C}^{4}: \lambda_{C}^{2}: 1
$$

We find that the subleading order corrections to the charged lepton mass matrix will break the residual symmetry $Z_{3}^{T}$ but they are suppressed by $\lambda_{C}^{2}$ with respect to LO results, thus can be safely neglected.

In the neutrino sector, the lowest dimensional operators responsible for neutrino masses are

$$
w_{\nu}=\frac{y_{a}}{\Lambda}\left(L \phi_{a}\right)_{\mathbf{1}} H_{u} \nu_{\mathrm{atm}}^{c}+\frac{y_{s}}{\Lambda}\left(L \phi_{s}\right)_{\mathbf{1}^{\prime}} H_{u} \nu_{\mathrm{sol}}^{c}+\frac{x_{a}}{2} \nu_{\mathrm{atm}}^{c} \nu_{\mathrm{atm}}^{c} \xi_{a}+\frac{x_{s}}{2} \nu_{\mathrm{sol}}^{c} \nu_{\mathrm{sol}}^{c} \xi_{s},
$$

where the coupling constants $y_{a}, y_{s}, x_{a}$ and $x_{s}$ are restricted to be real by the imposed CP symmetry. Inserting the vacuum alignments in Eqs. $(59,66)$, we can read out the neutrino Dirac and Majorana mass matrices as follow,

$$
m_{D}=\left(\begin{array}{ccc}
0 & -y_{a} v_{\phi_{a}} & y_{a} v_{\phi_{a}} \\
y_{s} v_{\phi_{s}} & \frac{5}{2} y_{s} v_{\phi_{s}} & -\frac{1}{2} y_{s} v_{\phi_{s}}
\end{array}\right) \frac{v_{u}}{\Lambda}, \quad m_{N}=\left(\begin{array}{cc}
x_{a} v_{\xi_{a}} & 0 \\
0 & x_{s} v_{\xi_{s}}
\end{array}\right)
$$

with $v_{u}=\left\langle H_{u}\right\rangle$. Applying the seesaw formula, we obtain the light neutrino mass matrix $m_{\nu}$ in Eq. (11) with

$$
m_{a}=\left|\frac{y_{a}^{2} v_{\phi_{a}}^{2}}{x_{a} v_{\xi_{a}}} \frac{v_{u}^{2}}{\Lambda^{2}}\right|, \quad m_{s}=\left|\frac{y_{s}^{2} v_{\phi_{s}}^{2}}{x_{s} v_{\xi_{s}}} \frac{v_{u}^{2}}{\Lambda^{2}}\right| .
$$

Note that we have used the result shown in Eq. (70) under the assumption of $x_{a} x_{s}>0$. In the case

of $x_{a} x_{s}<0$, in order to obtain the desired value $\eta=-\pi / 2$, the phase of the ratio $\frac{v_{\phi_{s}}^{2} v_{\xi_{a}}}{v_{\phi_{a}}^{2} v_{\xi_{s}}}$ should be $\pi / 2$, i.e. we could choose the right side of Eq. (70) as $\pi / 2$. In short, the neutrino mass matrix of the new Littlest Seesaw model is realized exactly, hence the phenomenological predictions in 
section 2.1 follows immediately. The NLO contributions to the neutrino mass matrices in Eq. (77) are found to be suppressed by $\lambda_{C}^{2}$ and consequently we will not discuss them.

Similar to other discrete flavour symmetry models, the solution of the vacuum alignment problem requires complicated constructions in our model and some new superfields which are SM singlets are introduced, as shown above. Recently it was suggested that the complexity of the vacuum alignment problem can be reduced if modular invariance plays the role of flavour symmetry [76]. In particular, we find that $\operatorname{CSD}(n)$ model with $n=1+\sqrt{6}$ can be naturally obtained if the VEV of the complex modulus $\tau$ is at certain fixed point [77]. We expect that the desired alignment corresponding to $x=-1 / 2$ can also be reproduced from some modular group, such that our model could be simplified considerably.

\subsection{Charged lepton flavour violating radiative decays}

In the following, we shall present the predictions for charged lepton flavour violating (LFV) radiative decays. It is usually assumed that the SUSY breaking mechanism is flavour blind at some high energy scale. In the minimal supergravity scenario, the slepton mass matrices are diagonal and universal in flavour and the trilinear couplings are proportional to the Yukawa couplings at the GUT scale. Non-vanishing off-diagonal elements are generated in both the slepton mass matrices and the trilinear couplings because of the renormalization group running effect at low energy, leading to charged lepton flavour violation processes induced in SUSY models. In the mass insertion and leading log approximations, the branching ratio of the charged lepton LFV radiative decay is given to good approximation by $[78,79]$

$$
B r\left(l_{i} \rightarrow l_{j} \gamma\right) \simeq \frac{\alpha^{3}}{G_{F}^{2} m_{s}^{8}} \operatorname{Br}\left(l_{i} \rightarrow l_{j} \bar{\nu}_{j} \nu_{i}\right)\left|\left(m_{\tilde{L}}^{2}\right)_{i j}\right|^{2} \tan ^{2} \beta,
$$

where $G_{F}$ is the Fermi coupling constant and $m_{s}$ is the characteristic mass scale of the SUSY particle in the loop with

$$
m_{s}^{8} \simeq 0.5 m_{0}^{2} M_{1 / 2}^{2}\left(m_{0}^{2}+0.6 M_{1 / 2}^{2}\right)^{2} .
$$

The slepton doublet mass squared $m_{\tilde{L}}^{2}$ arises from the renormalization group evolution. To an excellent approximation, the renormalization group result has the form

$$
\left(m_{\tilde{L}}^{2}\right)_{i \neq j} \simeq-\frac{1}{8 \pi^{2}}\left(3 m_{0}^{2}+A_{0}^{2}\right)\left(\lambda^{\dagger} L \lambda\right)_{i j},
$$

where $\lambda$ is the neutrino Yukawa coupling matrix given in Eq. (30) and the factor $L$ is defined as

$$
L=\operatorname{diag}\left(\log \frac{M_{G}}{M_{1}}, \log \frac{M_{G}}{M_{2}}\right),
$$

with the GUT scale $M_{G} \simeq 2 \times 10^{16} \mathrm{GeV}$. For our model, we find the expressions of $\left(m_{\tilde{L}}^{2}\right)_{i \neq j}$ are as follows,

$$
\begin{aligned}
& \left(m_{\tilde{L}}^{2}\right)_{21} \simeq-\frac{5|b|^{2}\left(A_{0}^{2}+3 m_{0}^{2}\right)}{16 \pi^{2}} \log \left(\frac{M_{G}}{M_{2}}\right) \\
& \left(m_{\tilde{L}}^{2}\right)_{31} \simeq \frac{|b|^{2}\left(A_{0}^{2}+3 m_{0}^{2}\right)}{16 \pi^{2}} \log \left(\frac{M_{G}}{M_{2}}\right) \\
& \left(m_{\tilde{L}}^{2}\right)_{32} \simeq \frac{A_{0}^{2}+3 m_{0}^{2}}{32 \pi^{2}}\left[4|a|^{2} \log \left(\frac{M_{G}}{M_{1}}\right)+5|b|^{2} \log \left(\frac{M_{G}}{M_{2}}\right)\right] .
\end{aligned}
$$

As shown in Eq. (52), the right-handed neutrino mass $M_{1}$ is fixed to be $3.992 \times 10^{10} \mathrm{GeV}$ by leptogenesis. The best fit value of $r \equiv m_{s} / m_{a}$ is 0.145 in our model, consequently it is natural to 
take $M_{2} \simeq 3 \times 10^{11} \mathrm{GeV}$. The measured values of the lepton mixing angles and neutrino masses fix $m_{s}=|b|^{2} v_{u}^{2} / M_{2}=3.243 \mathrm{meV}$ which leads to $b \simeq 5.783 \times 10^{-3}$. For typical values of the soft SUSY breaking parameters $m_{0}=140 \mathrm{GeV}, M_{1 / 2}=600 \mathrm{GeV}, A_{0}=0$ and $\tan \beta=5$, we find the branching ratios of the charged lepton flavour violating radiative decays to be

$$
B r(\mu \rightarrow e \gamma) \simeq 1.745 \times 10^{-16}, \quad B r(\tau \rightarrow e \gamma) \simeq 1.244 \times 10^{-18}, \quad B r(\tau \rightarrow \mu \gamma) \simeq 2.647 \times 10^{-17},
$$

which are safely below the present experimental upper limits [80].

\subsection{UV completion}

In our model, we see that all interactions are renormalizable except the the charged lepton Yukawa couplings in Eq. (73) and the neutrino Yukawa couplings in Eq. (76). In the following, we shall give a UV completion which gives rise to these non-renormalizable operators upon integrating the heavy messengers fields. In order to generate the high dimensional operators relevant for charged lepton masses in Eq. (73), we introduce three pairs of messenger fields $\Sigma_{i}$ and $\Sigma_{i}^{c}$ with $i=1,2,3$ which transform under the flavour symmetry $S_{4} \times Z_{5} \times Z_{8}$ as follows

$$
\begin{array}{rlrl}
\Sigma_{1} & \sim(\mathbf{3}, 1,1), & \Sigma_{1}^{c} & \sim(\mathbf{3}, 1,1), \\
\Sigma_{2} & \sim\left(\mathbf{3}^{\prime}, \omega_{5}, \omega_{8}^{7}\right), \quad & \Sigma_{2}^{c} & \sim\left(\mathbf{3}^{\prime}, \omega_{5}^{4}, \omega_{8}\right), \\
\Sigma_{3} & \sim\left(\mathbf{3}, \omega_{5}^{2},-i\right), \quad \Sigma_{3}^{c} \sim\left(\mathbf{3}, \omega_{5}^{3}, i\right) .
\end{array}
$$

The chiral superfields $\Sigma_{i}$ and $\Sigma_{i}^{c}$ are singlets under the standard model gauge group and they carry hypercharges $Y=-1$ and $Y=1$ respectively, and their $U(1)_{R}$ charges are all +1 . The renormalizable terms containing these messenger fields read as

$$
\begin{aligned}
w_{l}^{\mathrm{UV}}= & g_{1}\left(L \Sigma_{1}^{c}\right)_{\mathbf{1}} H_{d}+g_{2}\left(\Sigma_{1} \phi_{l}\right)_{\mathbf{1}} \tau^{c}+g_{3}\left(\left(\Sigma_{1} \Sigma_{2}^{c}\right)_{\mathbf{3}} \phi_{l}\right)_{\mathbf{1}}+g_{4}\left(\Sigma_{2} \phi_{l}\right)_{\mathbf{1}^{\prime}} \mu^{c}+g_{5}\left(\left(\Sigma_{2} \Sigma_{3}^{c}\right)_{\mathbf{3}} \phi_{l}\right)_{\mathbf{1}} \\
& +g_{6}\left(\Sigma_{3} \phi_{l}\right)_{\mathbf{1}} e^{c}+M_{\Sigma_{1}}\left(\Sigma_{1} \Sigma_{1}^{c}\right)_{\mathbf{1}}+M_{\Sigma_{2}}\left(\Sigma_{2} \Sigma_{2}^{c}\right)_{\mathbf{1}}+M_{\Sigma_{3}}\left(\Sigma_{3} \Sigma_{3}^{c}\right)_{\mathbf{1}}
\end{aligned}
$$

where all the couplings $g_{1,2,3,4,5,6}$ and masses $M_{\Sigma_{1,2,3}}$ are fixed to be real by the CP symmetry. Integrating out the heavy messenger fields, we obtain the desired higher-dimensional operators in the effective theory,

$w_{l}^{\mathrm{eff}}=-\frac{g_{1} g_{2}}{M_{\Sigma_{1}}}\left(L \phi_{l}\right)_{\mathbf{1}} \tau^{c} H_{d}+\frac{g_{1} g_{3} g_{4}}{M_{\Sigma_{1}} M_{\Sigma_{2}}}\left(L\left(\phi_{l} \phi_{l}\right)_{\mathbf{3}^{\prime}}\right)_{\mathbf{1}^{\prime}} \mu^{c} H_{d}-\frac{g_{1} g_{3} g_{5} g_{6}}{M_{\Sigma_{1}} M_{\Sigma_{2}} M_{\Sigma_{3}}}\left(\left(L \phi_{l}\right)_{\mathbf{3}^{\prime}}\left(\phi_{l} \phi_{l}\right)_{\mathbf{3}^{\prime}}\right)_{\mathbf{1}} e^{c} H_{d}$,

which leads to a diagonal and hierarchical charged lepton mass matrix for the alignment of $\phi_{l}$ in Eq. (57). The non-renormalizable neutrino Dirac couplings in Eq. (76) can be generated with the help of the following heavy fields

$$
\begin{array}{ll}
\Sigma_{\nu_{1}} \sim(\mathbf{1}, 1,-i), \quad \Sigma_{\nu_{1}}^{c} \sim(\mathbf{1}, 1, i), \\
\Sigma_{\nu_{2}} \sim\left(\mathbf{1}^{\prime}, 1, \omega_{8}^{3}\right), \quad \Sigma_{\nu_{2}}^{c} \sim\left(\mathbf{1}^{\prime}, 1, \omega_{8}^{5}\right),
\end{array}
$$

which are all standard model doublets with hypercharge $Y= \pm \frac{1}{2}\left(-\right.$ for $\Sigma_{\nu_{i}}$ and + for $\left.\Sigma_{\nu_{i}}^{c}\right)$. The relevant terms in the UV completion are given by

$$
\begin{aligned}
w_{\nu}^{\mathrm{UV}}= & k_{1}\left(L \phi_{a}\right)_{1} \Sigma_{\nu_{1}}^{c}+k_{2} \Sigma_{\nu_{1}} \nu_{\mathrm{atm}}^{c} H_{u}+k_{3}\left(L \phi_{s}\right)_{1^{\prime}} \Sigma_{\nu_{2}}^{c}+k_{4} \Sigma_{\nu_{2}} \nu_{\mathrm{sol}}^{c} H_{u} \\
& +M_{\Sigma_{\nu_{1}}} \Sigma_{\nu_{1}} \Sigma_{\nu_{1}}^{c}+M_{\Sigma_{\nu_{2}}} \Sigma_{\nu_{2}} \Sigma_{\nu_{2}}^{c},
\end{aligned}
$$

where CP invariance requires the parameters $k_{1,2,3,4}$ and $M_{\Sigma_{\nu_{1,2}}}$ are real. Integrating out $\Sigma_{\nu_{1,2}}$ and $\Sigma_{\nu_{1,2}}^{c}$, we reproduce the desired terms

$$
w_{\nu}^{\mathrm{eff}}=-\frac{k_{1} k_{2}}{M_{\Sigma_{\nu_{1}}}}\left(L \phi_{a}\right)_{1} H_{u} \nu_{\mathrm{atm}}^{c}-\frac{k_{3} k_{4}}{M_{\Sigma_{\nu_{2}}}}\left(L \phi_{s}\right)_{1^{\prime}} H_{u} \nu_{\mathrm{sol}}^{c}
$$




\section{Conclusion}

In this paper we have proposed and discussed a new Littlest Seesaw model, realized in the tri-direct $\mathrm{CP}$ approach, in which the couplings of the two right-handed neutrinos to the lepton doublets are proportional to $(0,-1,1)$ and $(1,5 / 2,-1 / 2)$ respectively with the relative phase $\eta=-\pi / 2$. We have shown that this model can give an excellent description of lepton flavour mixing, including an atmospheric neutrino mixing angle in the second octant, in terms of only two input parameters. We also showed that the observed baryon asymmetry can be generated for the lightest right-handed neutrino mass $M_{1}=1.176 \times 10^{11} \mathrm{GeV}$ in SM and $M_{1}=3.992 \times 10^{10} \mathrm{GeV}$ in MSSM with $\tan \beta=5$. The model is based on the flavour symmetry $S_{4} \times Z_{5} \times Z_{8}$ in which the desired alignments and the phase $\eta=-\pi / 2$ are achieved.

We emphasise that the model independent tri-direct $\mathrm{CP}$ approach is a quite predictive scheme for constructing neutrino mass models based on discrete flavour symmetry and CP symmetry, even without specialising to a particular choice of the two real input parameters $\eta$ and $x$. Here we have focussed on the $\mathcal{N}_{1}$ case where the flavour symmetry $S_{4}$ and CP are broken to $Z_{3}^{T}$ in the charged lepton sector, $Z_{2}^{U} \times H_{C P}^{\text {atm }}$ in the atmospheric sector and $Z_{2}^{S U} \times H_{C P}^{\text {sol }}$ in the solar neutrino sector with $H_{C P}^{\text {atm }}=\{1, U\}$ and $H_{C P}^{\text {sol }}=\{1, S U\}$, the vacuum alignment of $\phi_{\text {atm }}$ and $\phi_{\text {sol }}$ would be fixed to $\left\langle\phi_{\text {atm }}\right\rangle \propto(0,1,-1)^{T}$ and $\left\langle\phi_{\text {sol }}\right\rangle \propto(1, x, 2-x)^{T}$, where importantly $x$ is real due to the residual $\mathrm{CP}$ symmetry. As a consequence, the lepton mixing matrix is determined to be the TM1 pattern, and the experimental data on neutrino mixing can be described very well. Thus the structure is enforced by residual symmetry in tri-direct CP approach, with $S_{4}$ flavour symmetry yielding good agreement with the present data for many examples, which include both the original Littlest Seesaw model and the new Littlest Seesaw model $[58,59]$.

It is interesting to compare the new Littlest Seesaw with $(x, \eta)=(-1 / 2,-\pi / 2)$ to the original Littlest Seesaw model with $(x, \eta)=(3,2 \pi / 3),(-1,-2 \pi / 3)[18,20,22]$, which also provides a good fit to the data, as summarized in table 1 . However we find that the new Littlest Seesaw with arguably simpler values $x=-1 / 2, \eta=-\pi / 2$, can provide a better description to the experimental data than the original Littlest Seesaw. The mixing parameters are predicted to lie in quite narrow regions, and they are all within the reach of future neutrino experiments. The denominator of the phase $\eta=-\pi / 2$ is the smallest one among the different benchmark values in table 1, consequently the case of $x=-1 / 2, \eta=-\pi / 2$ might be expected to be easier to realize in a concrete model than the original Littlest Seesaw and other cases listed in table 1.

We emphasise that the choice $x=-1 / 2$ and $\eta=-\pi / 2$ of the new Littlest Seesaw model, is both simpler and more successful than the original Littlest Seesaw model. As usual, all three lepton mixing angles, leptonic CP violation phases and three neutrino masses $\left(m_{1}=0\right)$ only depend on two input parameters $m_{a}$ and $r=m_{s} / m_{a}$ whose values can be determined by the precisely measured neutrino mass squared differences $\Delta m_{21}^{2}$ and $\Delta m_{31}^{2}$. The comprehensive numerical analysis shows that all lepton mixing parameters and neutrino masses are restricted in rather narrow regions, as shown in Eq. (17). The new Littlest Seesaw differs most markedly in its predictions for $\theta_{23}$ and $\delta_{C P}$. While the atmospheric mixing angle $\theta_{23}$ is predicted to be close to maximal in the original Littlest Seesaw model, it is predicted to be in the second octant and close to the current central value [8] in the new Littlest Seesaw model. Furthermore, we have extended the new Littlest Seesaw to $3 \mathrm{RHN}$ models in the section 2.2. In the $3 \mathrm{RHN}$ model, we obtain a smaller $\chi_{\text {min }}^{2}$ than the new Littlest Seesaw, and we find that the 2RHN model is a good approximation of the 3RHN model. Therefore our new Littlest Seesaw with $2 \mathrm{RHN}$ can be regarded as a decoupling limit of the $3 \mathrm{RHN}$ model.

The "maximal" phase $\eta=-\pi / 2$ is the unique source of CP violation in the new Littlest Seesaw model, as usual controlling both low energy $\mathrm{CP}$ violation and the $\mathrm{CP}$ asymmetry in leptogenesis. Hence the $\mathrm{CP}$ violation which may be observed in neutrino oscillations is related to the baryon asymmetry of the Universe. We have studied the generation of the baryon asymmetry of the 
Universe through leptogenesis in the new Littlest Seesaw model. We have numerically solved the flavoured Boltzmann equations for the lepton asymmetries, and found that the observed excess of matter over antimatter can be produced for the lightest right-handed neutrino mass $M_{1}=$ $1.176 \times 10^{11} \mathrm{GeV}$ in $\mathrm{SM}$ and $M_{1}=3.992 \times 10^{10} \mathrm{GeV}$ in MSSM with $\tan \beta=5$. We conclude that the new Littlest Seesaw model can give an excellent fit to the neutrino oscillation data and leptogenesis simultaneously.

Finally we have constructed a fully working explicit model based on the flavour group $S_{4}$ and CP symmetry which fixes the values of $x=-1 / 2$ and $\eta=-\pi / 2$ in the new Littlest Seesaw model. The charged lepton mass hierarchy is naturally realized in our model, and the required vacua $\left\langle\phi_{a}\right\rangle \propto(0,1,-1)^{T},\left\langle\phi_{s}\right\rangle \propto(1,-1 / 2,5 / 2)^{T}$ and the relative phase $\eta=-\pi / 2$ are readily generated through the supersymmetric $F$-term alignment mechanism. Furthermore, we have studied the predictions for the charged lepton radiative decays $\mu \rightarrow e \gamma, \tau \rightarrow e \gamma$ and $\tau \rightarrow \mu \gamma$, and have found that the resulting branch ratios are below the current experimental upper bounds. We have also presented a UV completion which gives rise to the non-renormalizable operators upon integrating out the heavy messenger fields.

It would be interesting to extend this predictive new Littlest Seesaw model to the quark sector to give a unified description of quark and lepton flavour mixing, for instance in the framework of a supersymmetric grand unified theory. We expect that the quark mass matrices would be related to the construction of the new Littlest Seesaw model in the lepton sector. This is left for future work.

\section{Acknowledgements}

P.-T. C. and G.-J. D. acknowledge the support of the National Natural Science Foundation of China under Grant Nos 11522546 and 11835013. S.F. K. acknowledges the STFC Consolidated Grant ST/L000296/1 and the European Union's Horizon 2020 research and innovation programme under the Marie Skłodowska-Curie grant agreements Elusives ITN No. 674896 and InvisiblesPlus RISE No. 690575. C.-C. L. is supported by National Natural Science Foundation of China under Grant No 11847228, China Postdoctoral Science Foundation Grant Nos. 2017M620258 and 2018T110617, CPSF-CAS Joint Foundation for Excellent Postdoctoral Fellows No. 2017LH0003, the Fundamental Research Funds for the Central Universities under Grant No. WK2030040090, the Anhui Province Natural Science Foundation Grant No. 1908085QA24 and the CAS Center for Excellence in Particle Physics (CCEPP).

\section{Appendix}

\section{A Group Theory of $S_{4}$}

In the present work, we adopt the same convention for the $S_{4}$ flavour symmetry group as [27, 29]. The $S_{4}$ group is generated by three generators $S, T$ and $U$ which obey the relations

$$
S^{2}=T^{3}=U^{2}=(S T)^{3}=(S U)^{2}=(T U)^{2}=(S T U)^{4}=1 .
$$

The group $S_{4}$ has 24 elements and five irreducible representations: $\mathbf{1}, \mathbf{1}^{\prime}, \mathbf{2}, \mathbf{3}$ and $\mathbf{3}^{\prime}$. The representation matrices of the three generators in different irreducible representations are chosen to be the following form

$$
\begin{array}{ccll}
\mathbf{1}, \mathbf{1}^{\prime}: & S=1, & T=1, & U= \pm 1, \\
\mathbf{2}^{\prime}: & S=\left(\begin{array}{ll}
1 & 0 \\
0 & 1
\end{array}\right), & T=\left(\begin{array}{cc}
\omega & 0 \\
0 & \omega^{2}
\end{array}\right), & U=\left(\begin{array}{ll}
0 & 1 \\
1 & 0
\end{array}\right), \\
\mathbf{3}, \mathbf{3}^{\prime}: & S=\frac{1}{3}\left(\begin{array}{ccc}
-1 & 2 & 2 \\
2 & -1 & 2 \\
2 & 2 & -1
\end{array}\right), & T=\left(\begin{array}{ccc}
1 & 0 & 0 \\
0 & \omega^{2} & 0 \\
0 & 0 & \omega
\end{array}\right), & U=\mp\left(\begin{array}{lll}
1 & 0 & 0 \\
0 & 0 & 1 \\
0 & 1 & 0
\end{array}\right),
\end{array}
$$




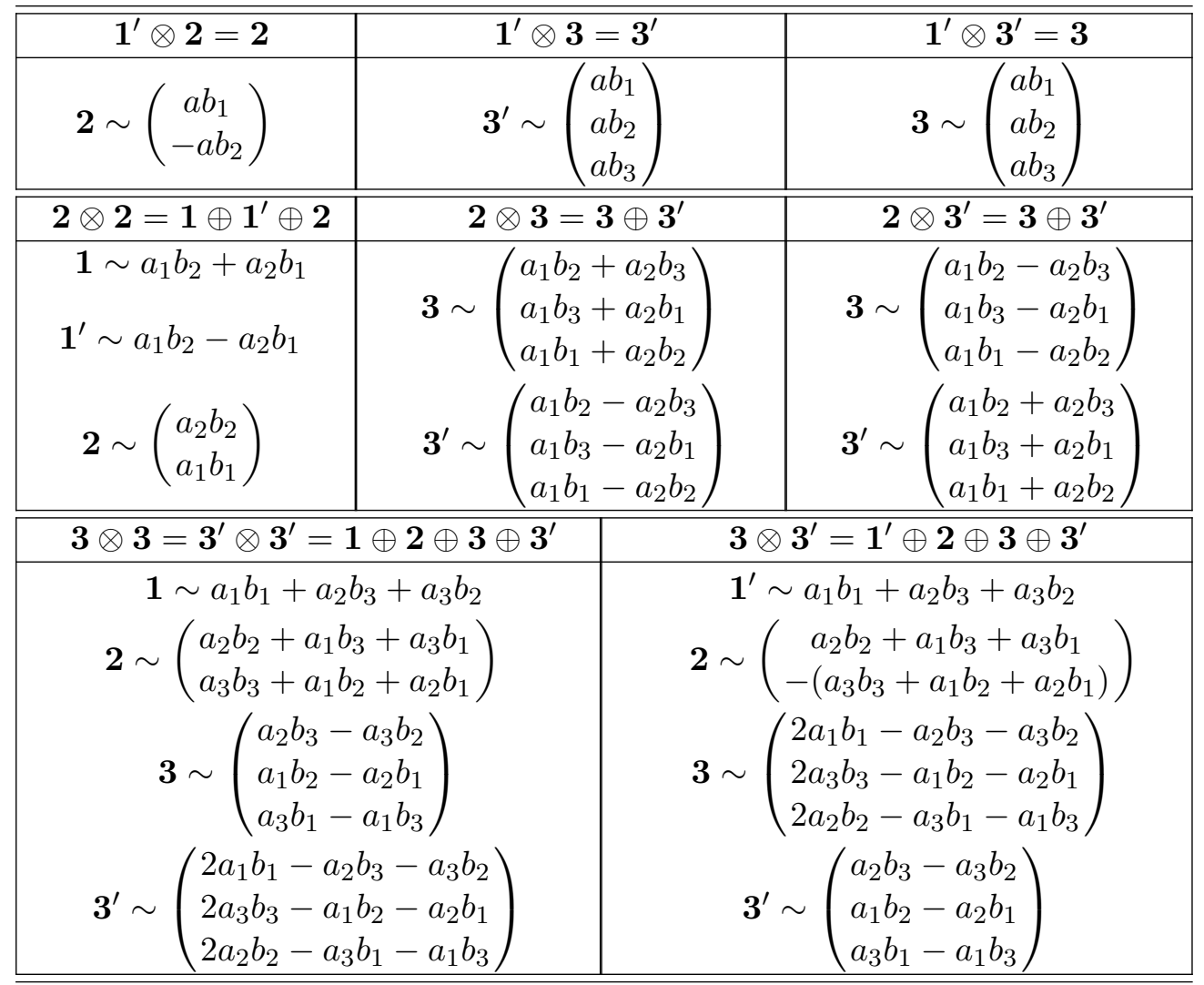

Table 3: The Kronecker products and Clebsch-Gordan coefficients of $S_{4}$ group [27, 29]. We use $a_{i}$ to indicate the elements of the first representation of the product and $b_{i}$ to indicate those of the second representation.

with $\omega=e^{2 \pi i / 3}$. As has been shown in [27,29], the generalized CP transformation compatible with the $S_{4}$ flavour symmetry is of the same form as the flavour symmetry transformation in our working basis,

$$
X_{\mathbf{r}}=\rho_{\mathbf{r}}(g), \quad g \in S_{4},
$$

where $g$ can be any of the 24 group elements of $S_{4}$. The $S_{4}$ Clebsch-Gordan coefficients are frequently used when building a model based on $S_{4}$ flavour symmetry. We summarise the Kronecker products and Clebsch-Gordan coefficients in our basis in table 3.

\section{References}

[1] S. F. King and C. Luhn, "Neutrino Mass and Mixing with Discrete Symmetry," Rept. Prog. Phys. 76 (2013) 056201, arXiv:1301.1340 [hep-ph].

[2] S. F. King, "Models of Neutrino Mass, Mixing and CP Violation," J. Phys. G42 (2015) 123001, arXiv:1510.02091 [hep-ph]. 
[3] P. Minkowski, " $\mu \rightarrow e \gamma$ at a Rate of One Out of $10^{9}$ Muon Decays?," Phys. Lett. 67 B (1977) 421-428.

[4] R. N. Mohapatra and G. Senjanovic, "Neutrino Mass and Spontaneous Parity Nonconservation," Phys. Rev. Lett. 44 (1980) 912. [,231(1979)].

[5] J. Schechter and J. W. F. Valle, "Neutrino Masses in SU(2) x U(1) Theories," Phys. Rev. D22 (1980) 2227.

[6] S. F. King, "Atmospheric and solar neutrinos with a heavy singlet," Phys. Lett. B439 (1998) 350-356, arXiv:hep-ph/9806440 [hep-ph].

[7] S. F. King, "Atmospheric and solar neutrinos from single right-handed neutrino dominance and U(1) family symmetry," Nucl. Phys. B562 (1999) 57-77, arXiv:hep-ph/9904210 [hep-ph] .

[8] I. Esteban, M. C. Gonzalez-Garcia, A. Hernandez-Cabezudo, M. Maltoni, and T. Schwetz, "Global analysis of three-flavour neutrino oscillations: synergies and tensions in the determination of $\theta_{23}, \delta_{C P}$, and the mass ordering," JHEP 01 (2019) 106, arXiv:1811.05487 [hep-ph] .

[9] S. F. King, "Large mixing angle MSW and atmospheric neutrinos from single right-handed neutrino dominance and U(1) family symmetry," Nucl. Phys. B576 (2000) 85-105, arXiv:hep-ph/9912492 [hep-ph].

[10] P. H. Frampton, S. L. Glashow, and T. Yanagida, "Cosmological sign of neutrino CP violation," Phys. Lett. B548 (2002) 119-121, arXiv:hep-ph/0208157 [hep-ph].

[11] S. F. King, "Constructing the large mixing angle MNS matrix in seesaw models with right-handed neutrino dominance," JHEP 09 (2002) 011, arXiv:hep-ph/0204360 [hep-ph].

[12] W.-l. Guo, Z.-z. Xing, and S. Zhou, "Neutrino Masses, Lepton Flavor Mixing and Leptogenesis in the Minimal Seesaw Model," Int. J. Mod. Phys. E16 (2007) 1-50, arXiv:hep-ph/0612033 [hep-ph].

[13] K. Harigaya, M. Ibe, and T. T. Yanagida, "Seesaw Mechanism with Occam's Razor," Phys. Rev. D86 (2012) 013002, arXiv:1205.2198 [hep-ph].

[14] J. Zhang and S. Zhou, "A Further Study of the Frampton-Glashow-Yanagida Model for Neutrino Masses, Flavor Mixing and Baryon Number Asymmetry," JHEP 09 (2015) 065, arXiv: 1505.04858 [hep-ph].

[15] S. F. King, "Predicting neutrino parameters from $\mathrm{SO}(3)$ family symmetry and quark-lepton unification," JHEP 08 (2005) 105, arXiv:hep-ph/0506297 [hep-ph].

[16] S. Antusch, S. F. King, C. Luhn, and M. Spinrath, "Trimaximal mixing with predicted $\theta_{13}$ from a new type of constrained sequential dominance," Nucl. Phys. B856 (2012) 328-341, arXiv:1108.4278 [hep-ph].

[17] S. F. King, "Minimal predictive see-saw model with normal neutrino mass hierarchy," JHEP 07 (2013) 137, arXiv:1304.6264 [hep-ph].

[18] S. F. King, "Littlest Seesaw," JHEP 02 (2016) 085, arXiv:1512.07531 [hep-ph].

[19] S. F. King and C. Luhn, "Littlest Seesaw model from $\mathrm{S}_{4} \times \mathrm{U}(1)$," JHEP 09 (2016) 023, arXiv:1607.05276 [hep-ph]. 
[20] P. Ballett, S. F. King, S. Pascoli, N. W. Prouse, and T. Wang, "Precision neutrino experiments vs the Littlest Seesaw," JHEP 03 (2017) 110, arXiv:1612.01999 [hep-ph].

[21] S. F. King, S. Molina Sedgwick, and S. J. Rowley, "Fitting high-energy Littlest Seesaw parameters using low-energy neutrino data and leptogenesis," JHEP 10 (2018) 184, arXiv:1808.01005 [hep-ph].

[22] S. F. King, "Minimal see-saw model predicting best fit lepton mixing angles," Phys. Lett. B724 (2013) 92-98, arXiv:1305.4846 [hep-ph].

[23] S. F. King, "A model of quark and lepton mixing," JHEP 01 (2014) 119, arXiv:1311.3295 [hep-ph].

[24] F. Björkeroth and S. F. King, "Testing constrained sequential dominance models of neutrinos," J. Phys. G42 no. 12, (2015) 125002, arXiv:1412.6996 [hep-ph].

[25] F. Feruglio, C. Hagedorn, and R. Ziegler, "Lepton Mixing Parameters from Discrete and CP Symmetries," JHEP 07 (2013) 027, arXiv:1211.5560 [hep-ph].

[26] M. Holthausen, M. Lindner, and M. A. Schmidt, "CP and Discrete Flavour Symmetries," JHEP 04 (2013) 122, arXiv:1211.6953 [hep-ph].

[27] G.-J. Ding, S. F. King, C. Luhn, and A. J. Stuart, "Spontaneous CP violation from vacuum alignment in $S_{4}$ models of leptons," JHEP 05 (2013) 084, arXiv:1303.6180 [hep-ph].

[28] G.-J. Ding, S. F. King, and A. J. Stuart, "Generalised CP and $A_{4}$ Family Symmetry," JHEP 12 (2013) 006, arXiv:1307.4212 [hep-ph].

[29] C.-C. Li and G.-J. Ding, "Generalised CP and trimaximal $T M_{1}$ lepton mixing in $S_{4}$ family symmetry," Nucl. Phys. B881 (2014) 206-232, arXiv:1312.4401 [hep-ph].

[30] G.-J. Ding and Y.-L. Zhou, "Predicting lepton flavor mixing from $\Delta(48)$ and generalized $C P$ symmetries," Chin. Phys. C39 no. 2, (2015) 021001, arXiv:1312.5222 [hep-ph].

[31] G.-J. Ding and S. F. King, "Generalized $C P$ and $\Delta(96)$ family symmetry," Phys. Rev. D89 no. 9, (2014) 093020, arXiv:1403.5846 [hep-ph].

[32] G.-J. Ding and Y.-L. Zhou, "Lepton mixing parameters from $\Delta(48)$ family symmetry and generalised CP," JHEP 06 (2014) 023, arXiv:1404.0592 [hep-ph].

[33] C.-C. Li and G.-J. Ding, "Deviation from bimaximal mixing and leptonic CP phases in $\mathrm{S}_{4}$ family symmetry and generalized CP," JHEP 08 (2015) 017, arXiv:1408.0785 [hep-ph].

[34] G.-J. Ding, S. F. King, and T. Neder, "Generalised CP and $\Delta\left(6 n^{2}\right)$ family symmetry in semi-direct models of leptons," JHEP 12 (2014) 007, arXiv:1409.8005 [hep-ph].

[35] P. Chen, C.-C. Li, and G.-J. Ding, "Lepton Flavor Mixing and CP Symmetry," Phys. Rev. D91 (2015) 033003, arXiv:1412.8352 [hep-ph].

[36] L. L. Everett, T. Garon, and A. J. Stuart, "A Bottom-Up Approach to Lepton Flavor and CP Symmetries," JHEP 04 (2015) 069, arXiv:1501.04336 [hep-ph].

[37] G. C. Branco, I. de Medeiros Varzielas, and S. F. King, "Invariant approach to CP in family symmetry models," Phys. Rev. D92 no. 3, (2015) 036007, arXiv:1502.03105 [hep-ph].

[38] C.-C. Li and G.-J. Ding, "Lepton Mixing in $A_{5}$ Family Symmetry and Generalized CP," JHEP 05 (2015) 100, arXiv:1503.03711 [hep-ph]. 
[39] A. Di Iura, C. Hagedorn, and D. Meloni, "Lepton mixing from the interplay of the alternating group $\mathrm{A}_{5}$ and CP," JHEP 08 (2015) 037, arXiv:1503.04140 [hep-ph].

[40] P. Ballett, S. Pascoli, and J. Turner, "Mixing angle and phase correlations from A5 with generalized CP and their prospects for discovery," Phys. Rev. D92 no. 9, (2015) 093008, arXiv:1503.07543 [hep-ph].

[41] G. C. Branco, I. de Medeiros Varzielas, and S. F. King, "Invariant approach to $\mathcal{C P}$ in unbroken $\Delta(27), "$ Nucl. Phys. B899 (2015) 14-36, arXiv:1505.06165 [hep-ph].

[42] P. Chen, C.-Y. Yao, and G.-J. Ding, "Neutrino Mixing from CP Symmetry," Phys. Rev. D92 no. 7, (2015) 073002, arXiv:1507.03419 [hep-ph].

[43] G.-J. Ding and S. F. King, "Generalized CP and $\Delta\left(3 n^{2}\right)$ Family Symmetry for Semi-Direct Predictions of the PMNS Matrix," Phys. Rev. D93 (2016) 025013, arXiv:1510.03188 [hep-ph].

[44] P. Chen, G.-J. Ding, F. Gonzalez-Canales, and J. W. F. Valle, "Generalized $\mu-\tau$ reflection symmetry and leptonic CP violation," Phys. Lett. B753 (2016) 644-652, arXiv:1512.01551 [hep-ph].

[45] C.-C. Li, C.-Y. Yao, and G.-J. Ding, "Lepton Mixing Predictions from Infinite Group Series $D_{9 n, 3 n}^{(1)}$ with Generalized CP," JHEP 05 (2016) 007, arXiv:1601.06393 [hep-ph].

[46] P. Chen, G.-J. Ding, and S. F. King, "Leptogenesis and residual CP symmetry," JHEP 03 (2016) 206, arXiv:1602.03873 [hep-ph].

[47] C.-Y. Yao and G.-J. Ding, "CP Symmetry and Lepton Mixing from a Scan of Finite Discrete Groups," Phys. Rev. D94 no. 7, (2016) 073006, arXiv:1606.05610 [hep-ph].

[48] C.-C. Li, J.-N. Lu, and G.-J. Ding, " $A_{4}$ and CP symmetry and a model with maximal CP violation," Nucl. Phys. B913 (2016) 110-131, arXiv:1608.01860 [hep-ph].

[49] J.-N. Lu and G.-J. Ding, "Alternative Schemes of Predicting Lepton Mixing Parameters from Discrete Flavor and CP Symmetry," Phys. Rev. D95 no. 1, (2017) 015012, arXiv:1610.05682 [hep-ph].

[50] L. L. Everett and A. J. Stuart, "Lepton Sector Phases and Their Roles in Flavor and Generalized CP Symmetries," Phys. Rev. D96 no. 3, (2017) 035030, arXiv:1611.03020 [hep-ph].

[51] C.-C. Li and G.-J. Ding, "Implications of residual CP symmetry for leptogenesis in a model with two right-handed neutrinos," Phys. Rev. D96 no. 7, (2017) 075005, arXiv:1701.08508 [hep-ph].

[52] C.-C. Li, J.-N. Lu, and G.-J. Ding, "Toward a unified interpretation of quark and lepton mixing from flavor and CP symmetries," JHEP 02 (2018) 038, arXiv:1706.04576 [hep-ph].

[53] J.-N. Lu and G.-J. Ding, "Quark and lepton mixing patterns from a common discrete flavor symmetry with a generalized CP symmetry," Phys. Rev. D98 no. 5, (2018) 055011, arXiv:1806.02301 [hep-ph].

[54] J.-N. Lu and G.-J. Ding, "Dihedral flavor group as the key to understand quark and lepton flavor mixing," JHEP 03 (2019) 056, arXiv:1901.07414 [hep-ph]. 
[55] P. Chen, S. Centelles Chuli, G.-J. Ding, R. Srivastava, and J. W. F. Valle, "Neutrino Predictions from Generalized CP Symmetries of Charged Leptons," JHEP 07 (2018) 077, arXiv: 1802.04275 [hep-ph].

[56] C. Hagedorn and E. Molinaro, "Flavor and CP symmetries for leptogenesis and $0 \nu \beta \beta$ decay," Nucl. Phys. B919 (2017) 404-469, arXiv:1602.04206 [hep-ph].

[57] L. A. Delgadillo, L. L. Everett, R. Ramos, and A. J. Stuart, "Predictions for the Dirac CP-Violating Phase from Sum Rules," Phys. Rev. D97 no. 9, (2018) 095001, arXiv:1801.06377 [hep-ph].

[58] G.-J. Ding, S. F. King, and C.-C. Li, "Tri-Direct CP in the Littlest Seesaw Playground," JHEP 12 (2018) 003, arXiv:1807.07538 [hep-ph].

[59] G.-J. Ding, S. F. King, and C.-C. Li, "Lepton Mixing Predictions from $S_{4}$ in the Tri-Direct CP approach to Two Right-handed Neutrino Models," arXiv:1811.12340 [hep-ph].

[60] G.-J. Ding, S. F. King, and C.-C. Li, "Golden Littlest Seesaw," Nucl. Phys. B925 (2017) 470-499, arXiv:1705.05307 [hep-ph].

[61] C. Jarlskog, "Commutator of the Quark Mass Matrices in the Standard Electroweak Model and a Measure of Maximal CP Violation," Phys. Rev. Lett. 55 (1985) 1039.

[62] G. C. Branco, L. Lavoura, and M. N. Rebelo, "Majorana Neutrinos and CP Violation in the Leptonic Sector," Phys. Lett. B180 (1986) 264-268.

[63] Planck Collaboration, N. Aghanim et al., "Planck 2018 results. VI. Cosmological parameters," arXiv:1807.06209 [astro-ph.CO].

[64] M. Fukugita and T. Yanagida, "Baryogenesis Without Grand Unification," Phys. Lett. B174 (1986) 45-47.

[65] E. Nardi, Y. Nir, E. Roulet, and J. Racker, "The Importance of flavor in leptogenesis," JHEP 01 (2006) 164, arXiv:hep-ph/0601084 [hep-ph].

[66] A. Abada, S. Davidson, A. Ibarra, F. X. Josse-Michaux, M. Losada, and A. Riotto, "Flavour Matters in Leptogenesis," JHEP 09 (2006) 010, arXiv:hep-ph/0605281 [hep-ph].

[67] A. Abada, S. Davidson, F.-X. Josse-Michaux, M. Losada, and A. Riotto, "Flavor issues in leptogenesis," JCAP 0604 (2006) 004, arXiv:hep-ph/0601083 [hep-ph].

[68] S. Antusch, S. F. King, and A. Riotto, "Flavour-Dependent Leptogenesis with Sequential Dominance," JCAP 0611 (2006) 011, arXiv:hep-ph/0609038 [hep-ph].

[69] G. F. Giudice, A. Notari, M. Raidal, A. Riotto, and A. Strumia, "Towards a complete theory of thermal leptogenesis in the SM and MSSM," Nucl. Phys. B685 (2004) 89-149, arXiv:hep-ph/0310123 [hep-ph].

[70] W. Buchmuller, P. Di Bari, and M. Plumacher, "Leptogenesis for pedestrians," Annals Phys. 315 (2005) 305-351, arXiv:hep-ph/0401240 [hep-ph].

[71] L. Covi, E. Roulet, and F. Vissani, "CP violating decays in leptogenesis scenarios," Phys. Lett. B384 (1996) 169-174, arXiv:hep-ph/9605319 [hep-ph].

[72] W. Buchmuller, R. D. Peccei, and T. Yanagida, "Leptogenesis as the origin of matter," Ann. Rev. Nucl. Part. Sci. 55 (2005) 311-355, arXiv:hep-ph/0502169 [hep-ph]. 
[73] S. Davidson, E. Nardi, and Y. Nir, "Leptogenesis," Phys. Rept. 466 (2008) 105-177, arXiv:0802.2962 [hep-ph].

[74] J. A. Harvey and M. S. Turner, "Cosmological baryon and lepton number in the presence of electroweak fermion number violation," Phys. Rev. D42 (1990) 3344-3349.

[75] G. Altarelli and F. Feruglio, "Tri-bimaximal neutrino mixing, A(4) and the modular symmetry," Nucl. Phys. B741 (2006) 215-235, arXiv:hep-ph/0512103 [hep-ph].

[76] F. Feruglio, "Are neutrino masses modular forms?," in From My Vast Repertoire ...: Guido Altarelli's Legacy, A. Levy, S. Forte, and G. Ridolfi, eds., pp. 227-266. 2019. arXiv:1706.08749 [hep-ph].

[77] G.-J. Ding, S. F. King, X.-G. Liu, and J.-N. Lu, "Modular $\mathrm{S}_{4}$ and $\mathrm{A}_{4}$ symmetries and their fixed points: new predictive examples of lepton mixing," JHEP 12 (2019) 030, arXiv:1910.03460 [hep-ph].

[78] F. Borzumati and A. Masiero, "Large Muon and electron Number Violations in Supergravity Theories," Phys. Rev. Lett. 57 (1986) 961.

[79] J. Hisano, T. Moroi, K. Tobe, and M. Yamaguchi, "Lepton flavor violation via right-handed neutrino Yukawa couplings in supersymmetric standard model," Phys. Rev. D53 (1996) 2442-2459, arXiv:hep-ph/9510309 [hep-ph].

[80] Particle Data Group Collaboration, M. Tanabashi et al., "Review of Particle Physics," Phys. Rev. D98 no. 3, (2018) 030001. 